\title{
Celecoxib Treatment Improves Neurologic Deficit and Reduces Selective Neuronal Loss and Glial Response in Rats after Transient Middle Cerebral Artery Occlusion
}

\author{
María Santos-Galdiano, Diego Pérez-Rodríguez, Berta Anuncibay-Soto, \\ Enrique Font-Belmonte, Irene F. Ugidos, Carlos César Pérez-García, \\ and Arsenio Fernández-López
}

\begin{abstract}
Área de Biología Celular, Instituto de Biomedicina (M.S.-G., D.P.-R., B.A.-S., E.F.-B., I.F.U., A.F.-L.) and Departamento de Medicina, Cirugía y Anatomía Veterinaria (C.C.P.-G.), Universidad de León and Neural Therapies SL, Edificio Institutos de Investigación (B.A.-S.), León, Spain
\end{abstract}

Received June 8, 2018; accepted October 4, 2018

\begin{abstract}
Areas of selective neuronal loss (SNL) represent the first morphologic signs of damage in the penumbra region and are considered putative targets for ischemic stroke therapy. We performed a novel assessment of measuring the effects of the anti-inflammatory agent celecoxib by analyzing simultaneously the different neural populations (neurons, astrocytes, and microglia cells) in SNL and non-SNL areas. Rats were subjected to 1 hour of middle cerebral artery occlusion (MCAO) and treated with celecoxib 1 and 24 hours after ischemia. Infarct volume measurements and triple immunostaining of neurons (neuronal nuclear antigen), microglia (ionized calcium-binding adaptor molecule 1), and astroglia were performed after 12 and 48 hours
\end{abstract}

of reperfusion. Motor response was tested by standard behavioral assays at $3,12,24$, and 48 hours. Confocal analysis revealed that the percentage of SNL areas, microglia densities, and glial activation increased at 48 hours of reperfusion. Celecoxib treatment improved the neurologic deficit, reduced the infarct volume by $50 \%$ after 48 hours of reperfusion, and resulted in a reduced percentage of SNL areas and microglia and astroglia reactivity after 48 hours of reperfusion. This study proves, for the first time, that celecoxib presents postischemic neuroprotective effects in a transient MCAO model, prevents or delays the presence of SNL areas, and reduces glial activation.

\section{Introduction}

Stroke is a major cause of death and the primary reason for permanent disability in developed countries (Creutzfeldt et al., 2012); occlusion of the middle cerebral artery (MCAO) or its branches accounts for approximately $70 \%$ of human ischemic strokes (Fluri et al., 2015). The rodent model of MCAO is widely accepted as a representative of human ischemic stroke and is used extensively to study potential treatments against stroke (Block et al., 2005). In this model, two different ischemic areas are defined: 1) the ischemic core, with severe loss of blood flow, which involves early cell death and typically affects the striatum; and 2) the surrounding area, penumbra, with mild blood flow loss, which will involve

This study was supported by Junta de Castilla y León (Grant LE025P17) and MINECO and FEDER funds (reference RTC-2015-4094-1), which also support M.S.-G. I.F.U. is granted by the Junta de Castilla y León (Grant EDU/ 310/2015). Enrique Font-Belmonte is supported by the University of León. Neural Therapies SL provided additional financial support.

https://doi.org/10.1124/jpet.118.251264. delayed, progressive infarction and typically affects the dorsolateral cortex (Carmichael, 2005; Heit and Wintermark, 2016).

After stroke, inflammatory reaction is responsible for damage progression and is the main mechanism of cell degeneration in the penumbra (Barone and Feuerstein, 1999; Wang et al., 2007). Inflammation involves the activation of microglia and astrocytes and infiltration of leukocytes into the brain (Taylor and Sansing, 2013). Microglia have a dual role after ischemic insult. On the one hand, microglia play a neuroprotective role that involves the production of neurotrophic factors and serve as scavenger cells. On the other hand, microglia cells release cytokines and reactive oxygen species, leading to brain edema and neuronal damage (Shichita et al., 2012). In physiologic conditions, microglia present small somata with an extensive arborization of dynamic processes (Walker et al., 2014). After ischemic insult, microglia are rapidly activated, and the branch number and the length of their processes are progressively attenuated

ABBREVIATIONS: Ab, antibody; ANOVA, analysis of variance; BSA, bovine serum albumin; C, contralateral; COX, cyclooxygenase; Cx, cerebral cortical area; DAPI, 4,6-diamino-2-phenylindole; FrPaSS, frontoparietal cortex somatosensory area; GFAP, glial fibrillary acidic protein; I, injured; IBA-1, ionized calcium- binding adaptor molecule 1; ICH, intracerebral hemorrhage; I/R, ischemia/reperfusion; MCA, middle cerebral artery; MCAO, middle cerebral artery occlusion; NeuN, neuronal nuclear antigen; NSAID, nonsteroidal anti-inflammatory drugs; PBS, phosphate-buffered saline; PFA, paraformaldehyde; Pir, piriform cortex; SNL, selective neuronal loss; TFI, total fluorescence intensity; tMCAO, transient middle cerebral artery occlusion; TTC, 2,3,5-triphenyltetrazolium chloride. 
until an ameboid morphology is achieved (Yenari et al., 2010; Karperien et al., 2013), mirroring the severity of ischemic damage (Ito et al., 2001). Astrocyte reactivity includes hypertrophy of cell soma and processes, as well as upregulation of intermediate filaments. Thus, glial fibrillary acidic protein (GFAP) and astrocyte swelling have been reported to increase after stroke (Wilhelmsson et al., 2006; Barreto et al., 2011).

Ischemic lesions can be classified as either selective neuronal loss (SNL), partial infarction, or pan-necrosis (Ejaz et al., 2013). SNL is defined as the death of single neurons with preserved extracellular matrix and tissue bulk, which appear in the penumbra area after transient MCAO (tMCAO) (Heiss and Rosner, 1983; García et al., 1996; Emmrich et al., 2015). There is a growing interest in SNL as the primary phase of ischemic lesion (Baron et al., 2014) because is possible that it impedes and delays behavioral recovery and, therefore, might represent a new therapeutic target of ischemic stroke (Baron, 2005).

Many pharmacologic strategies focus on limiting the postischemic inflammatory reaction to control damage progression (Shichita et al., 2012; Galea and Brough, 2013). Strong evidence supports a prominent role for cyclooxygenase 2 (COX2) in cerebral ischemic injury (Candelario-Jalil and Fiebich, 2008). Genetic deletion of COX2 reduces ischemic cell death in both focal and global cerebral ischemia models (Nogawa et al., 1997; Araki et al., 2001), whereas chronic overexpression of COX2 increases ischemic brain damage (Doré et al., 2003). Additionally, selective pharmacologic inhibition of COX2 with nonsteroidal anti-inflammatory drugs (NSAIDs) improves neurologic outcome after stroke (Nogawa et al., 1997; Candelario-Jalil et al., 2002; Wang et al., 2014). Classic NSAIDs, such as nonselective COX inhibitors, have a high frequency of gastrointestinal adverse effects (Shientag et al., 2012; Altman et al., 2015), and pharmaceutical companies have developed preferential selective antiCOX2 drugs, such as the "coxib" family, which are considered safer anti-inflammatory agents (Moore et al., 2005); however, some adverse effects associated with increased risk of ischemic stroke have been reported for some of these agents, such as rofecoxib and etoricoxib (Andersohn et al., 2006; Chang et al., 2010; Varas-Lorenzo et al., 2011).

Celecoxib has 375 times higher selectivity for COX2 compared with COX1 (Vane et al., 1998) and no or very low correlation with the risk of increased stroke (Andersohn et al., 2006). This drug presents neuroprotective properties when used in intracerebral hemorrhage (ICH) (Sinn et al., 2007; Lee et al., 2013; Chu et al., 2004) and is one of most potent NSAIDs in attenuating cell death in oxygen and glucose deprivation assays performed in brain slices (López-Villodres et al., 2012).

Here, we describe, for the first time, the neuroprotective effect of postischemic celecoxib treatment in a rat tMCAO model. We analyzed the progression of SNL areas and its histologic correlation with inflammation, characterizing the glial response (astroglia and microglia) in SNL and non-SNL areas.

\section{Materials and Methods}

Animals. We used 109 male Sprague-Dawley rats, aged 8 weeks (weight, 330-360 g). Only rats that showed a clear decrease in cerebral blood flow of MCA when the filament was introduced and returned to normal flow when the filament was removed were included in the study. Using these criteria, five animals were excluded from the assay. Six other rats died from the surgery, and two rats were excluded since they did not show ischemic damage. Therefore, only 96 met the conditions for the analysis. Animals were caged in pairs and housed at $22 \pm 1^{\circ} \mathrm{C}$ and under a 12:12 hour light/dark cycle, with food (Panlab, Barcelona, Spain) and water ad libitum. All experimental procedures were approved by the scientific committee of the University of León and performed in accordance with the Animal Research: Reporting of In Vivo Experiments (ARRIVE) guidelines and the Guidelines of the European Union Council (2010/63/EU) according to Spanish regulation (RD53/2013) for the use of laboratory animals. All efforts were made to minimize animal suffering and reduce the number of animals used.

The animals were randomly chosen for surgery; the person who performed the surgery was not the same person who assigned the treatment. For the dose-response assays of neuroprotection, we checked saline (as vehicle) and administered celecoxib, 20, 50, 100, 200 , and $400 \mathrm{mg} / \mathrm{kg}$. Each condition was assayed in 10 animals (60 animals total). In these animals, the protective effect was tested by 2,3,5-triphenyltetrazolium chloride (TTC) 48 hours after ische$\mathrm{mia} /$ reperfusion $(\mathrm{I} / \mathrm{R})$. In addition, in these animals, the neurologic score was measured at $0,3,12,24$, and 48 hours after I/R. An additional group and its respective control group were used to test the determined optimal celecoxib dose $(100 \mathrm{mg} / \mathrm{kg})$ at 12 hours of $\mathrm{I} / \mathrm{R}$ (20 animals total).

Four additional groups comprising four animals each were used for the immunocytochemical assays. Animals of one group were administered a single celecoxib dose ( $100 \mathrm{mg} / \mathrm{kg}) 1$ hour after ischemia, and animals in its corresponding control group were administered saline only. Both groups were euthanized and fixed by perfusion at 12 hours of I/R. Animals of the third group were administered two doses of $100 \mathrm{mg} / \mathrm{kg}$ celecoxib 1 and 24 hours after ischemia, and the fourth group (control) was administered saline only. The third and fourth groups were euthanized and fixed by perfusion 48 hours after I/R.

Focal Cerebral Ischemia. MCAO was induced as previously described (Ugidos et al., 2017). Briefly, anesthesia induction was performed in an anesthesia box using $3.5 \%-4 \%$ isoflurane in $\mathrm{O}_{2}$ enriched air with a flow of 2 liters $/ \mathrm{min}$. Then, animals were maintained anesthetized using $2 \%$ isoflurane in $\mathrm{O}_{2}$-enriched air with a flow of 2 liters/min through a facemask adapted to rats. Animals were maintained on a heating pad, and the temperature was monitored with a rectal probe and maintained at $37 \pm 0.5^{\circ} \mathrm{C}$. A Doppler probe (PeriFlux System 5000, Perimed AB, Stockholm, Sweden) was stuck on the temporal bone area located over the MCA to monitor the blood flow in this artery. After exposing the carotid bifurcation, a coated monofilament (cat. no. 403956PK5Re; Doccol Corporation, Sharon, MA) was inserted into the right common carotid artery and led through the right internal carotid artery until it blocked the origin of the MCA, which was tested with the Doppler probe. The filament was fixed with a suture in the common carotid artery; incisions were temporally closed with surgical staples, and animals recovered. One hour later, the animals were newly anesthetized under the same conditions, the monofilament was withdrawn, and the incisions were sutured. Reperfusion of blood was also monitored by the Doppler probe.

Celecoxib Treatment. Dose-response assays of celecoxib (Pfizer, New York) were made testing by the effect of 20, 50, 100, 200, and $400 \mathrm{mg} / \mathrm{kg}$. Celecoxib was dissolved in saline and administered intraperitoneally 1 hour after ischemia, and vehicle animals were injected with saline solution. Optimal efficacy for neuroprotection was established using a $100 \mathrm{mg} / \mathrm{kg}$-dose of celecoxib that was also used for the immunocytochemical study.

Neurologic Score. Vehicle and 20, 50, 100, 200, and $400 \mathrm{mg} / \mathrm{kg}$ celecoxib-treated animals were tested on each behavioral test at 0 (before MCAO-induced), 3, 12, 24, and 48 hours after I/R. Animal behavior was recorded and subsequently analyzed by two blinded observers. Neurologic deficits were scored using the scale previously described by Senda et al. (2011), which is summarized in Table 1 . The 
TABLE 1

Scale for neurologic score of middle cerebral artery occlusion-induced rats

A. Postural reflex

0 : When suspended by the tail, the animal extends both forelimbs toward the floor

1: Failure to extend the right forepaw fully, with or without wrist flexion

2: The animal flexes the right forepaw, and the shoulder touches the thorax

3: The animal rotates the body to the side of the affected forepaw, with a head-to-tail tendency

B. Circling motion

0: Normal, straight-line locomotion

1: Open circling to the right side, alternating with locomotion in a straight line

2: Open but persistent circling to the right

3: Closed and persistent circling (the animal turns consistently around its vertical axis, with the hindlimbs staying at the same place)

C. Falling to the contralateral side

0 : Absence of falling

1: Mild, apparent fall. The mandible does not touch the floor

2: Expressive fall (the right shoulder and mandible touch the floor)

D. Placement of the right forelimb during locomotion

0 : Normal (the rat can use the forelimb well, step-by-step)

1: Placement of the right forelimb is somewhat hesitant, as if the rat is walking with a "limp"

2: If the rat moves (circling), then the right forelimb is dragged and stays under the thorax

E. General state of alertness or consciousness

0 : The rat walks spontaneously and responds promptly to different stimuli (e.g., touch, sound, etc.)

1: If undisturbed, then the animal stays quiet but responds or moves when stimulated

2: The animal does not move at all, even when touched. It stays prostrated, apparently stuporous, and does not react when touched

sum of each behavioral test gave the total neurologic score, which was a maximum of 12 points, and 0 in noninjured rats. The final neurologic score was the average of the total score obtained by the two observers.

TTC Staining and Measurement of Infarct Volume. Animals used for the TTC technique were decapitated, and their brains were quickly removed and placed in a cold brain matrix for rats (ASI Instruments, Houston, TX) to obtain 2-mm- thick coronal brain sections. Infarct volume was assessed using the TTC method (Bederson et al., 1986), one of the most common histochemical stains used to assess cerebral injury. TTC is reduced in mitochondria, and viable tissue is stained red, whereas tissue with mitochondrial dysfunction remains colorless and represents the infarct region (Bederson et al., 1986). Briefly, sections were incubated in 1\% TTC in $50 \mathrm{mM}$ phosphate-buffered saline (PBS), $\mathrm{pH}$ 7.4, for 30 minutes at $37^{\circ} \mathrm{C}$ in darkness. Then, sections were fixed overnight in $4 \%$ paraformaldehyde (PFA) in $50 \mathrm{mM}$ PBS, $\mathrm{pH} \mathrm{7.4}$, at $4^{\circ} \mathrm{C}$ and digitized at 600 dpi resolution with a scanner (Canoscan LIDE 200; Canon Inc., Tokyo, Japan). Infarct volume was measured using ImageJ software (NIH) and calculated from the six sections that encompass the damaged area. Infarct volume is estimated as the percentage of total brain volume and adjusted for edema according to methods previously described by Reglodi et al. (2003). Briefly, edema index was calculated as the ratio/total volume of the ipsilateral hemisphere/total volume of the contralateral hemisphere. Thus, the percentage of infarct volume $=100 \times\left(\right.$ nonstained volume $\left(\mathrm{mm}^{3}\right) /$ edema index $) /$ total volume $\left(\mathrm{mm}^{3}\right)$.

Fixation and Processing of Tissue. For immunofluorescence assays, the rats were euthanized with an intraperitoneal injection of $200 \mathrm{mg} / \mathrm{kg}$ sodium pentobarbital. Animals were then transcardially perfused with $0.9 \% \mathrm{NaCl}$, followed by $4 \%$ PFA in PBS. Brains were removed, postfixed overnight in the $4 \%$ PFA in PBS, and then cryoprotected in $30 \%$ sucrose in PBS at $4^{\circ} \mathrm{C}$ until they sank. To make sectioning easier, the brains were embedded overnight in a 1:1 solution of Tissue Teck (Sakura Finetek, Torrance, CA) and 30\% sucrose in PBS and cut into $40-\mu \mathrm{m}$ - thick coronal slices with a freezing microtome (Microm HM450; Micron Technology, Inc., Boise, ID).

Immunofluorescence. Sections were incubated in $0.05 \%$ Tween20 in $10 \mathrm{mM}$ sodium citrate buffer, $\mathrm{pH} 6.0$, at $80^{\circ} \mathrm{C}$ for 30 minutes for epitope retrieval. Next, successive blocking of endogenous biotin with an avidin-biotin kit (SP-2001; Vector Laboratories, Inc., Burlingame, CA) and immunoglobulins with $1 \%$ bovine serum albumin (BSA) in $0.2 \%$ Triton X-100 was done in PBS at room temperature. Double staining for microglia and astroglia was done by incubating the sections overnight at $4^{\circ} \mathrm{C}$ in $1 \% \mathrm{BSA}, 0.2 \%$ Triton $\mathrm{X}-100$ in PBS with goat anti-IBA-1 (ionized calcium-binding adaptor molecule 1) polyclonal antibody (Ab) (1:2000) (ab5076; Abcam, Cambridge, MA) and rabbit anti-GFAP polyclonal antibody (1:500) (Z0334; Dako, Carpinteria, CA). A biotinylated anti-goat antibody (1:500) (BA9500; Vector Laboratories) was used to recognize anti-IBA-1 Ab and, in turn, was labeled with extravidin conjugated with alexa-647 (1:500) (S-2174; ThermoFisher Scientific). A goat anti-rabbit IgG conjugated with Alexa-488 (1:500) (A-11008; ThermoFisher Scientific, Waltham, MA) was used to recognize the anti-GFAP Ab. Afterward, sections were incubated overnight at $4^{\circ} \mathrm{C}$ in $1 \%$ BSA, $0.2 \%$ Triton $\mathrm{X}-100$ in PBS with mouse anti-NeuN monoclonal Ab (1:500) (MAB377, Millipore, Burlington, MA). This Ab was recognized with a goat anti-mouse IgG conjugated with Alexa-568 (1:500) (A-11004; Molecular Probes, Eugene, OR). Nuclei were counterstained with 4,6-diamidino-2phenylindole (DAPI) and sections mounted using Fluoromount G Mounting Medium (Life Technologies, Carlsbad, CA).

Image Acquisition. Animals perfused with 4\% PFA were used for image analysis. For each animal, seven equidistant $(960 \mu \mathrm{m})$ coronal brain sections from bregma $2.20 \mathrm{~mm}$ to bregma $-3.80 \mathrm{~mm}$ were used. Images, including IBA-1, GFAP, NeuN, and DAPI, stainings were obtained using a Zeiss LSM 800 confocal microscope and Zen Blue software. An image of each whole section with a resolution of 1.25 $\mu \mathrm{m} /$ pixel was obtained from tile scan images captured using the Tile Scan Module included in Zen Blue software and a Plan-Apochromat $10 \times / 0.45$ M27 objective (Carl Zeiss GmbH, Oberkochen, Germany). These images were used to measure SNL areas and the whole cortical area. Cell countings, as well as morphometric and densitometric analysis of glial populations, were performed using a modification of the optical dissector method (Gundersen et al., 1988). In brief, square optical dissectors of $0.255 \mathrm{~mm}^{2}$, separated $0.64 \mathrm{~mm}$ from each other, with a lateral resolution of $0.156 \mu \mathrm{m} /$ pixel, were programmed to be automatically obtained with a Plan-Apochromat $40 \times / 1$.3Oil DIC (UV) VIS-IR M27 (Carl Zeiss GmbH) along the frontoparietal cortex somatosensory area (FrPaSS) and piriform cortex (Pir) (Fig. 1A). Each dissector comprised seven levels in $z$-axis separated by $3 \mu \mathrm{m}(21 \mu \mathrm{m}$ in total). Laser power, detector gain, pinhole, and pixel dwell were kept constant for all the images to prevent bias in image quantification. Finally, obtained images were encoded with numbers to perform a blinded quantification.

SNL Area Quantification. SNL area quantification was carried out with Image $J$ software on the whole-cortex section images. The best approach to identify SNL is to use specific neuronal antibodies, such as NeuN (neuronal nuclear antigen), which stains only mature neurons; these SNLs are clearly visible as areas with weak staining and can be analyzed by counting the remaining neurons. SNL areas 

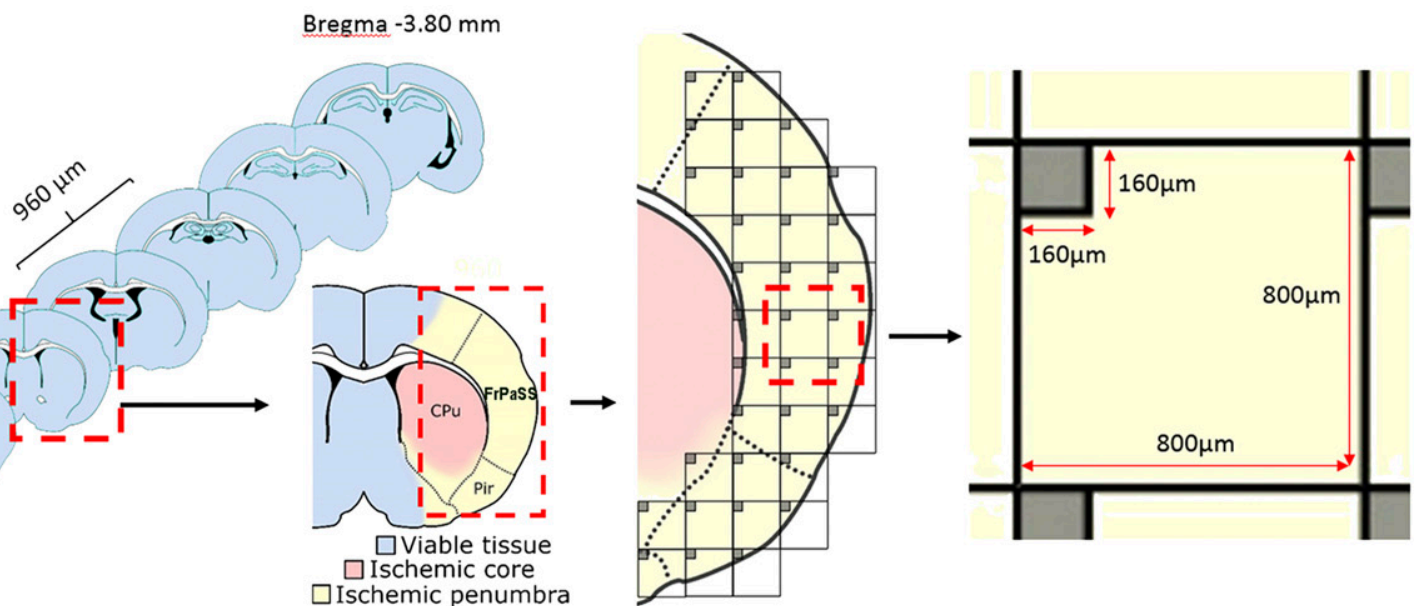

Bregma $2.20 \mathrm{~mm}$ $\square$ Ischemic penumbra

B

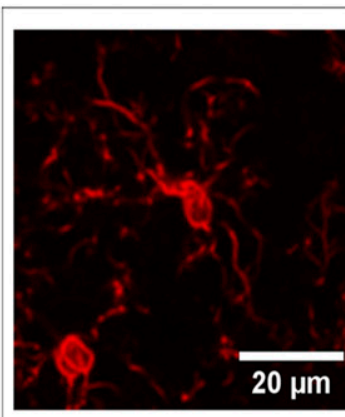

IBA-1 image
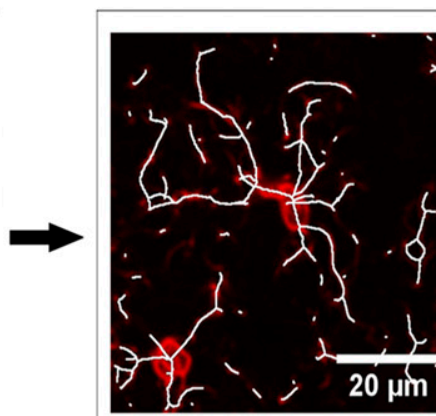

Segmenation and skeletonization

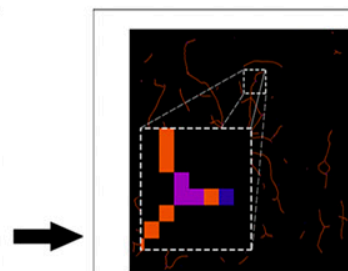

Endpoint voxel

Slab voxel

Branching voxel

\section{Skeleton analysis}

\section{Branch length/cell \\ Endpoints/cell \\ Number of cells}

\section{C}

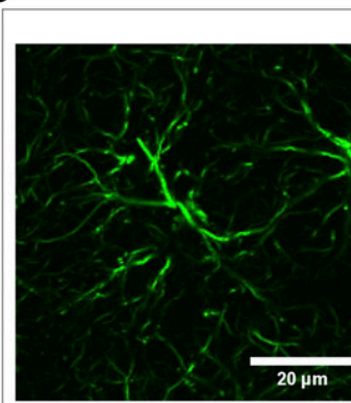

GFAP image
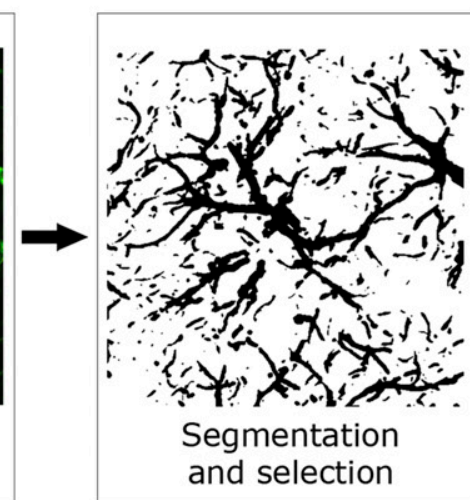
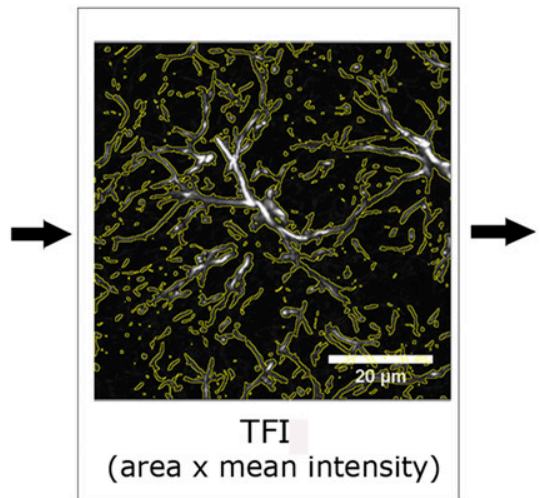

\section{TFI / cell Number of cells}

Fig. 1. Microscopy image analysis workflow. (A) Schematic representation of the sampling procedure for confocal acquisition. Seven equidistant coronal sections between bregma $2.20 \mathrm{~mm}$ and bregma $-3.80 \mathrm{~mm}$ cover $960 \mu \mathrm{m}$ were analyzed. Images from the whole section obtained from tiled scan images were used to define a grid of optical dissectors in FrPaSS and Pir regions from both the contralateral and injured hemisphere. A confocal microscope was programmed to acquire equidistant optical dissectors (gray squares) comprising seven images separated by $3 \mu \mathrm{m}$ along the $z$-axis, thus discarding about $9 \mu \mathrm{m}$ from the top and bottom of the 40- $\mu \mathrm{m}$-thick section. Dissectors were used for astroglia and microglia analysis, as well as for neuron counting. (B) Workflow used to measure microglia process branches and length. IBA-1 $z$-stack images were skeletonized by converting microglia processes in interconnected one-pixel-thick lines in the three dimensions. Skeleton analysis separates voxels (pixel 3) forming the lines in three types: slab voxels (orange) that connect two neighbor voxels, branching voxels (pink) that connect three or more neighbor voxels, (i.e., forming a ramification), and endpoint voxels that connect only one neighbor voxel, thus indicating the end of a process. (C) Workflow used to estimate astrocyte volume and GFAP amount. GFAP z-stack images were binarized to create a mask that was used to measure both the mean gray value of the GFAP image converted to grayscale and its area. This allows us to obtain TFI for each level (TFI). The TFI values for each of the seven images of the z-stack were summed and divided by the number of astrocyte nuclei to express the final results as TFIs/cell.

were outlined and measured. The total area corresponding to SNLs was divided by the area of the whole cortex of the injured hemisphere. Results were expressed as follows: percentage of SNL area $=100 \times$ ( $\Sigma$ SNL areas of the seven sections/ $/$ cortical area of injured hemisphere of the seven sections).
Microglia Activation Analysis. The number of IBA-1+ cells in each dissector was counted, and the average of the dissectors of each region was expressed as the number of microglia cells $/ \mathrm{mm}^{3}$. The branch number and the length of the cell processes have been used to quantify the degree of microglia activation. The summed process 
length and the number of process endpoints in each dissector were measured according to the method developed by Morrison and Filosa (2013), with minor modifications (Anuncibay-Soto et al., 2018). In brief, each z-stack image level, corresponding to IBA-1 staining, was binarized and then skeletonized using ImageJ. Skeleton analysis was carried out with Analyze Skeleton 2D/3D plugin in ImageJ (ArgandaCarreras et al., 2010). Results were normalized to the number of microglia nuclei per dissector, thus obtaining the averages of the number of process endpoints/cell and the summed process length/cell (Fig. 1B).

Astroglial Activation Analysis. In situations of severe astrogliosis, astrocytes proliferate and interlace their processes and loss of their individual domains (Sofroniew and Vinters, 2010). To quantify the degree of astroglial activation, we applied an image analysis method to measure both the volume of astrocytes and their GFAP protein levels (Anuncibay-Soto et al., 2018). The seven levels of each dissector corresponding to GFAP staining were converted to eight-bit grayscale images. These images were also binarized. Binary images were used to create a mask outlining each astrocyte soma and its processes, which was used to measure the whole area of the astrocyte. The mask of each level was used to select the corresponding gray image in each level and measure the mean gray value. The product of the mean gray value and the mask area is known as total fluorescence intensity (TFI). Thus, the sum of the seven TFIs of each dissector (one by level) was divided by the number of astroglial nuclei of the corresponding dissector and expressed as TFI/cell (Fig. 1C).
Statistical Analysis. Statistical analysis was performed using GraphPad Prism 6.0 Software (GraphPad Software, San Diego, CA). Normality test was assessed by the Shapiro-Wilk test. Kruskal-Wallis, followed by Dunn's test, was performed to analyze neurologic score. One-way ANOVA, followed by Tukey's test, was performed to analyze the infarct volume in the dose-response assay. Two-way ANOVA followed by Tukey's test was used in the rest of the assays. The level of significance was set at $P<0.05$. Our approach was to remove the outlier points by eliminating any points that were above (mean $+2 *$ S.D. and any points below (mean $-2 *$ S.D.).

\section{Results}

Celecoxib Reduces Neurologic Deficit after tMCAO. The SUM Summatory score of neurologic deficit increases along the time in vehicle animals. Different celecoxib doses, ranging from 20 to $400 \mathrm{mg} / \mathrm{kg}$ were assayed, and the $100-\mathrm{mg} /$ $\mathrm{kg}$ showed better reduction of neurologic deficit (Fig. 2A). This celecoxib dose can reduce the neurologic score to about three points at 12 hours after I/R and maintains this score until 48 hours after I/R (Fig. 2A).

Post-ischemic Celecoxib Treatment Reduces Infarct Volume after tMCAO. Caudate putamen appeared as the main infarcted area 12 hours after I/R, which represents about the $8 \%$ of the total brain volume. The infarcted area at

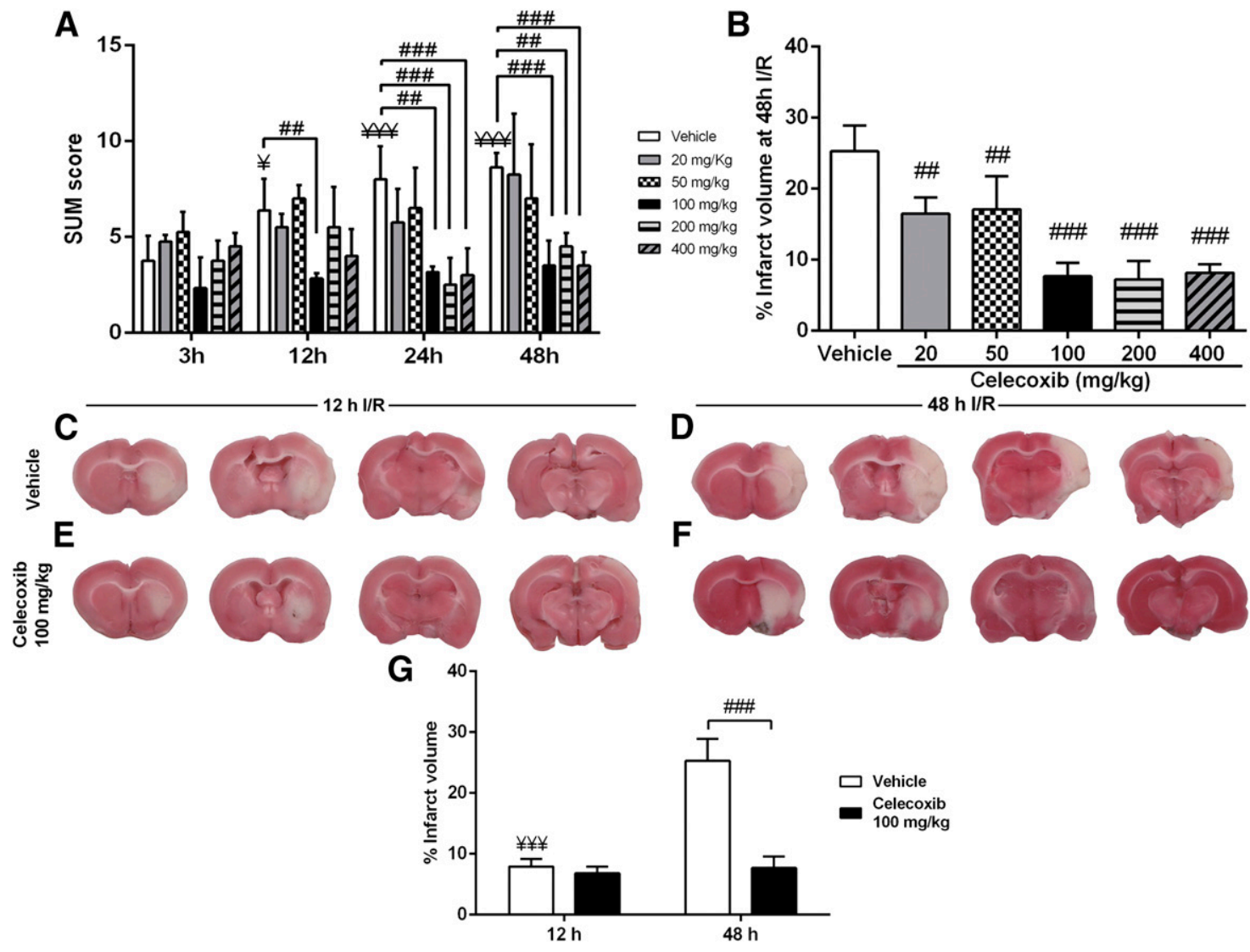

Fig. 2. Neurologic deficit and infarct volume measurement. (A) Celecoxib dose-dependent SUM score for neurologic deficit at $3,12,24$, and 48 hours of reperfusion (Kruskal-Wallis followed by Dunn's test, $n=10$ ). (B) Celecoxib dose-dependent infarct volume values (mean \pm S.E.M.); 48 hours after ischemia (one-way ANOVA, followed by Tukey's test, $n=10$ ). (C-F) Representative 2-mm-thick coronal brain slices, stained with TTC of (C and D) vehicle animals and ( $\mathrm{E}$ and $\mathrm{F}$ ) animals treated with $100 \mathrm{mg} / \mathrm{kg}$ celecoxib after 12 hours of I/R and 48 hours of I/R, respectively. (G) Percentage of infarct volume (mean \pm S.E.M.) in vehicle (white columns) and celecoxib-treated (black columns) animals (two-way ANOVA, followed by Tukey's test, $n=10$ ). ${ }^{\#}$ Significant differences from celecoxib treatment. ${ }^{¥}$ Time-dependent significant differences. One symbol, $P<0.05$; two symbols, $P<0.01$; three symbols, $P<0.001$. 
48 hours after I/R included caudate putamen, FrPaSS, and Pir and represented about the $25 \%$ of total brain volume (Fig. 2, C, $\mathrm{D}$, and $\mathrm{G}$ ).

Of the different celecoxib doses assayed, $100 \mathrm{mg} / \mathrm{kg}$ was identified as having the maximum neuroprotective effect (Fig. $2 B)$. Treatment with this celecoxib dose did not significantly modify the infarct volume 12 hours after I/R (Fig. 2, E and G); however, 48 hours after I/R, animals tha received $100 \mathrm{mg} / \mathrm{kg}$ of celecoxib showed an infarcted volume of about $11 \%$ of brain volume. This effect represented a reduction of $50 \%$ in the ischemic damage (Fig. 2, F and G).

SNL Area Increase with Time Is Prevented by Celecoxib. Significant neuronal density decreases in FrPaSS, but not in Pir, were observed in the injured (I) hemisphere 12 hours after I/R compared with their respective regions of contralateral (C) hemisphere; however, at 48 hours of reperfusion, the neuronal density of both I FrPaSS and Pir were significantly lower than their contralateral regions. The treatment with celecoxib resulted in a significant reduction of neuronal demise in these regions (Fig. 3, A and B).

SNLs were found from 12 hours of I/R in both I FrPaSS and Pir (Fig. 3C). At 12 hours of I/R, the area occupied by SNL represented about $4 \%$ of the whole cerebral cortical area $(\mathrm{Cx})$ of the I hemisphere. At 48 hours after I/R, this area increased significantly, reaching about $8 \%$ of the $\mathrm{Cx}$ of the I hemisphere. Treatment with celecoxib did not modify the SNL area percentage at 12 hours after $\mathrm{I} / \mathrm{R}$ but prevented the increase observed in the SNL area percentage 48 hours after $\mathrm{I} / \mathrm{R}$ in vehicle animals (Fig. 3, C and D).

The neuronal density of both SNL and non-SNL areas of I hemisphere were also quantified independently (Fig. 3, E and F). Neuronal density in the FrPaSS and Pir SNL areas was significantly lower compared with either the non-SNL areas or its corresponding contralateral region at both 12 and 48 hours after I/R. Non-SNL areas presented significant neuronal demise in FrPaSS, but not in Pir, compared with their respective contralateral regions at 12 and 48 hours after I/R. SNL and non-SNL areas did not reveal time-dependent differences. After 12 hours of reperfusion, we observed a significant attenuation of neuronal demise after treatment with celecoxib in FrPaSS SNL and non-SNL areas, as well as in Pir SNL areas; however, after 48 hours of reperfusion, a celecoxib effect on neuronal demise was only observed in Pir non-SNL areas.

Celecoxib Modulates tMCAO-Induced Microglia Activation. At 12 hours of reperfusion, we could not detect significant differences in IBA-1 + cell density resulting from ischemia in FrPaSS (Fig. 4A) or Pir (Fig. 4D); however, the ischemic insult resulted in significant decreases in length and number of microglia branches in both regions (Fig. 4, B, C, E, and F). At this time, these parameters were not modified by treatment with celecoxib.

After 48 hours of reperfusion, a significant increase in IBA$1+$ cell density, as well as a significant decrease in the length and number of microglia branches, were observed in vehicle I FrPaSS (Fig. 4, A-C) and Pir (Fig. 4, D-F) compared with their respective $\mathrm{C}$ regions; however, numerous changes were observed in FrPaSS but not in Pir. Thus, treatment with celecoxib significantly reduced the IBA- $1+$ cell density in FrPaSS, although this remained significantly higher than in C region (Fig. 4A). Also, in I FrPaSS, treatment with celecoxib significantly increased the number of branches (Fig. 4C) and showed a trend to increase the branch length (Fig. 4B) with respect to that observed in vehicle animals. In addition, in I FrPaSS, the number of microglia branches decreased at 48 hours of reperfusion compared with that observed after 12 hours of reperfusion (Fig. 4C).

IBA-1+ cell density, as well as length and number of branches of SNL and non-SNL areas of injured FrPaSS (Fig. 5) and Pir (Fig. 6), were also analyzed. After 12 hours of reperfusion, IBA-1+ cell densities and branching parameters were similar in FrPaSS SNL and non-SNL areas in both celecoxib treated and vehicle animals (Fig. 5). After 48 hours of reperfusion, however, some significant differences between SNL and non-SNL areas were observed. Thus, IBA-1+ cell density increased significantly in SNL compared with nonSNL areas, and both areas showed higher density than C FrPaSS (Fig. 5, A and D). In addition, in SNL areas, the branch number significantly decreased in 48I/R compared with 12I/R (Fig. 5, C and D). Celecoxib treatment decreased the IBA1+ cell density in only $48 \mathrm{I} / \mathrm{R}$ SNL areas consistently (Fig. 5, A and D) and branch number increases in both SNL and non-SNL areas at this time (Fig. 5, C and D). IBA-1positive cell densities or branching parameters were similar in Pir SNL and non-SNL areas, in both celecoxib treated and vehicle animals at both 12 and 48 hours after reperfusion (Fig. 6).

Celecoxib Modifies tMCAO-Induced Astroglial Reactivity but Not Density. Similar astroglia cell density values were observed in I FrPaSS (Fig. 7A) and Pir (Fig. 7C) compared with their respective $\mathrm{C}$ regions in all the conditions analyzed (12I/ $\mathrm{R}, 48 \mathrm{I} / \mathrm{R}$, vehicle and treated animals). TFI values showed a trend to be higher in both 12I/R I FrPaSS and Pir than in their respective $\mathrm{C}$ regions. These increases were significantly higher after 48 hours of reperfusion in both regions (Fig. 7, B and D). Treatment with celecoxib reduced the TFI I values after 48 hours of reperfusion in Pir, but these remained higher than for C Pir (Fig. 7D).

Astrocyte density and TFI were also measured independently in SNL and non-SNL areas of FrPaSS (Fig. 8) and Pir (Fig. 9). No significant changes in astrocyte density were observed in any of the conditions studied, either in FrPaSS (Fig. 8, A and C) or in Pir (Fig. 9, A and C). TFI values in 12I/R FrPaSS SNL areas of vehicle animals showed significant increases compared with its respective $\mathrm{C}$ region (Fig. 8, B and C); however, after 48 hours of reperfusion, both SNL and non-SNL areas of FrPaSS (Fig. 8, B and C) and Pir (Fig. 9, B and C) showed significantly higher TFI values compared with respective $\mathrm{C}$ region. Treatment with celecoxib was able to reduce TFI values only in Pir non-SNL areas after 48 hours of reperfusion (Fig. 9, B and C).

\section{Discussion}

The controversial effects of COX2 inhibitors has been extensively reviewed previously (Candelario-Jalil and Fiebich, 2008). Why these agents can result in different effects is not known, and more data are required to understand the mechanisms involved. Recent assays show that postischemic treatment with other NSAIDs in MCAO models reduce infarct volume (Bhattacharya et al., 2013; Ugidos et al., 2017) or apoptosis (Ye et al., 2013). This study demonstrates for the first time that postischemic celecoxib treatment has a neuroprotective effect in a rat tMCAO model. Our data are 
A

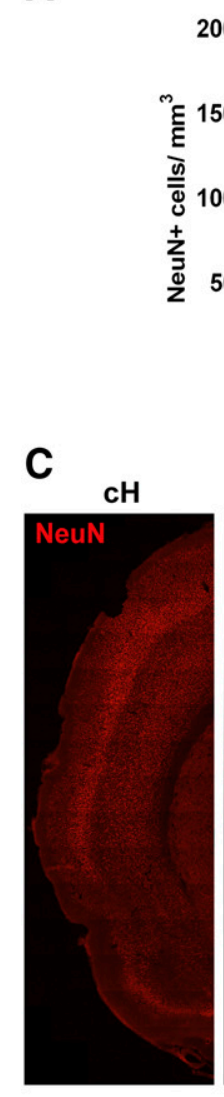

FrPaSS

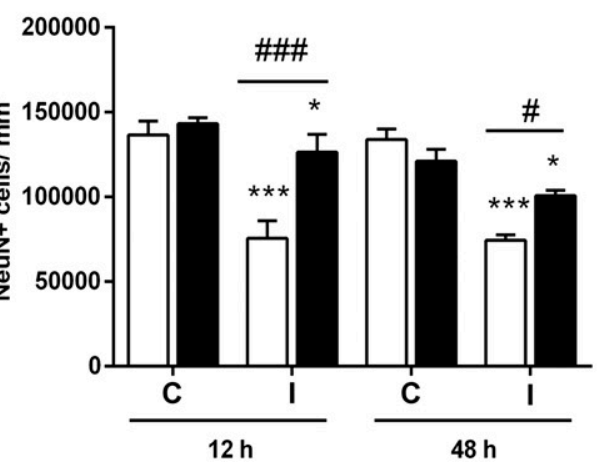

$12 \mathrm{~h}$ I/R Veh

$12 \mathrm{~h} \mathrm{I/R} \mathrm{Cel}$

48 h I/R Veh
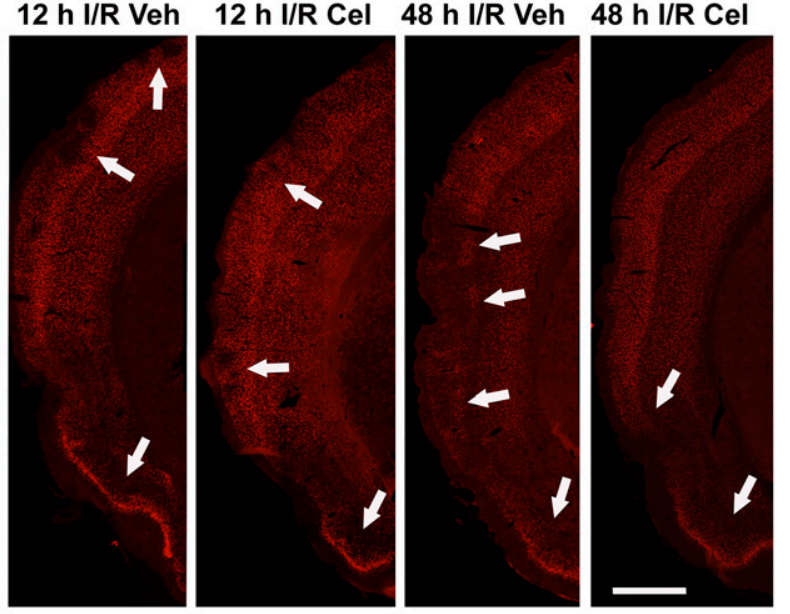

B

Pir

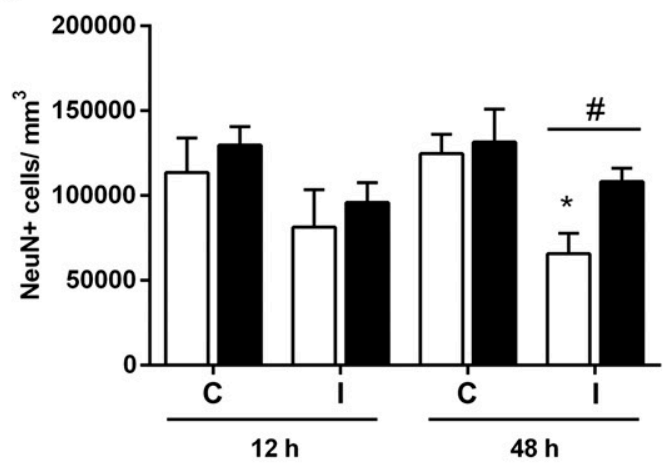

D

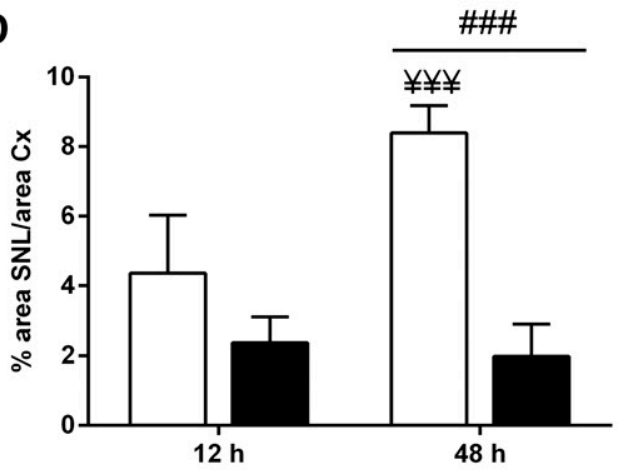

E

FrPaSS

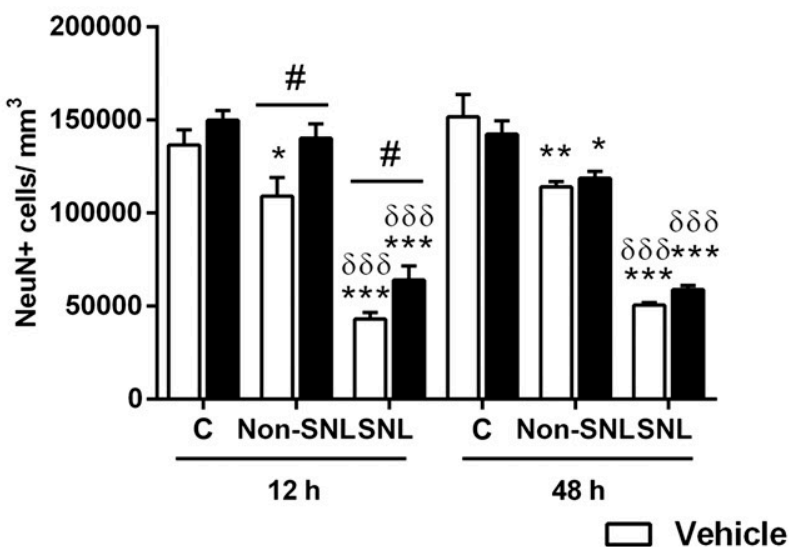

F

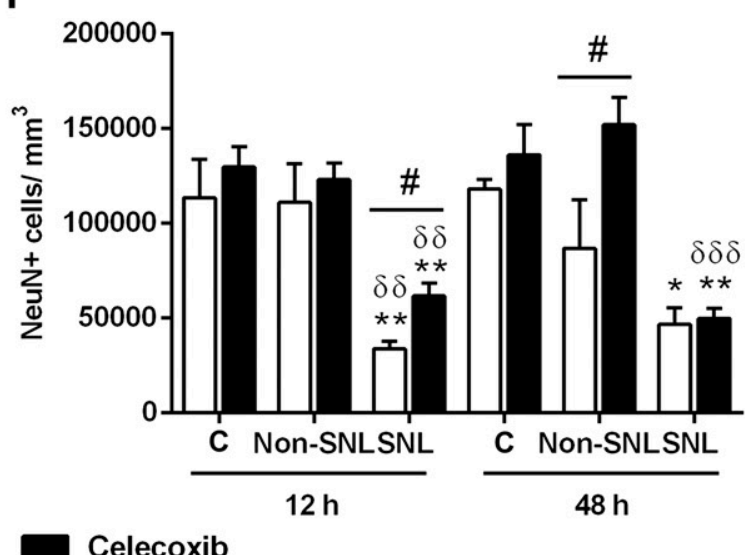

Celecoxib

Fig. 3. Neuronal demise after tMCAO. Neuronal densities (mean \pm S.E.M.) in contralateral (C) and injured (I) (A) frontoparietal cortex, somatosensory area (FrPaSS), and (B) piriform cortex (Pir) at 12 and 48 hours of reperfusion. (C) Representative images of the whole Cx area of the different conditions studied labeled with NeuN. Arrows point to areas of SNL. Scale bar, $1 \mathrm{~mm}$. (D) Percentage of SNL areas with respect to injured cortical area. Neuronal densities (mean \pm S.E.M.) in the C region, non-SNL and SNL areas of FrPaSS (E), and Pir (F) after 12 and 48 hours of reperfusion. Values of vehicle- and celecoxib-treated animals are shown in white and black columns, respectively. *Significant differences with respect to the contralateral region; ${ }^{*}$ Timedependent significant differences. One symbol represents $P<0.05$; two symbols, $P<0.01$; and three symbols, $P<0.001$. Two-way ANOVA followed by Tukey's test, $n=4$.

consistent with a previous study indicating that celecoxib administration before injury has a neuroprotective effect in a mouse permanent MCAO model (Ji and Tsirka, 2012). Interestingly, postischemic celecoxib treatment in ICH models has been described as having anti-inflammatory, analgesic, antihematoma, and antiedema effects (Sinn et al., 2007; Lee et al., 2013; Chu et al., 2014). To our knowledge, however, there are no reports on the postischemic treatments in
tMCAO. In principle, the basis of the neuroprotective effect of celecoxib could be supposed to rely on its anti-inflammatory effect; however, some of its properties make this hypothesis unappealing. In this regard, celecoxib induces different effects depending on the primary signal (Kaloustian et al., 2007). Thus, celecoxib behaves as an antitumoral agent in different cell lines exhibiting proapoptotic effects (Leahy et al., 2002; Schönthal, 2007; Pyrko et al., 2008), which has led to a wide 

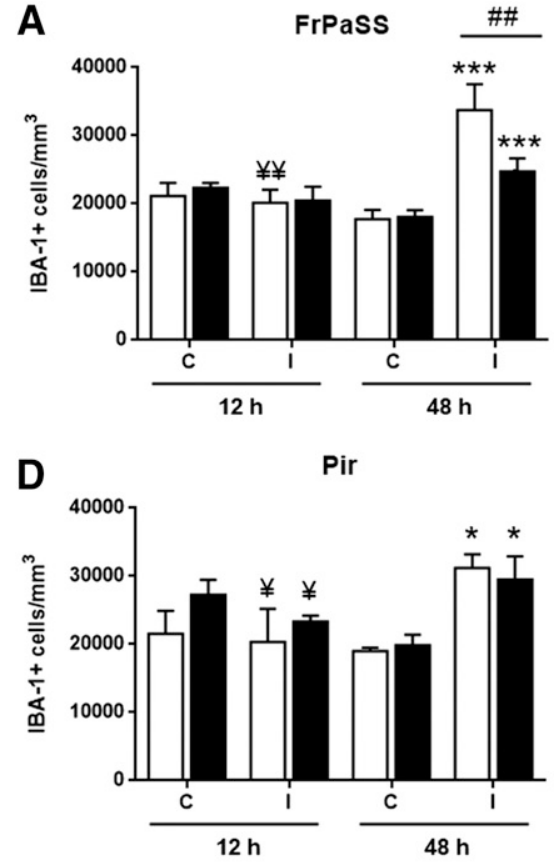

B

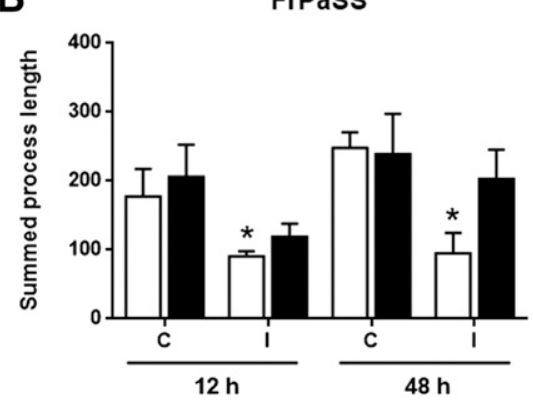

E

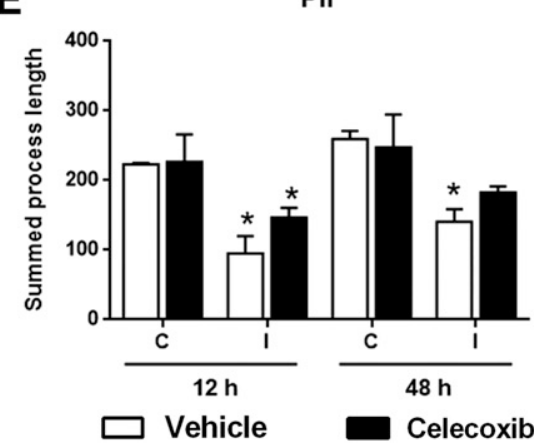

C

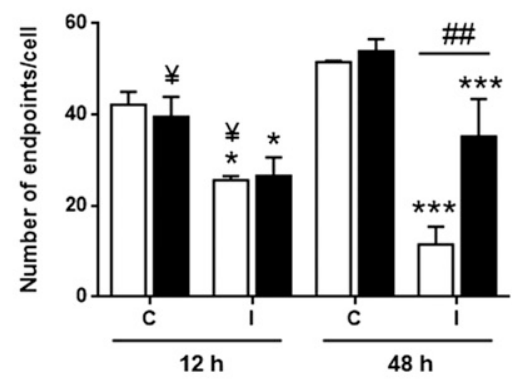

$\mathbf{F}$

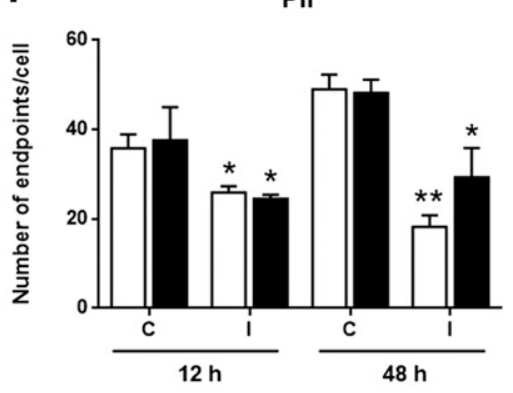

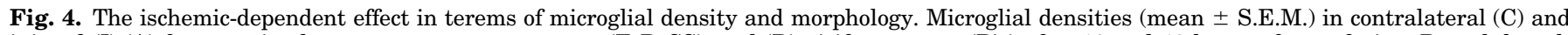

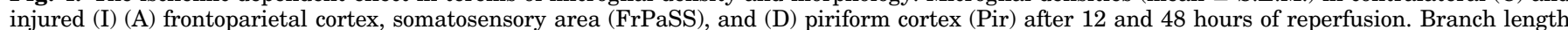

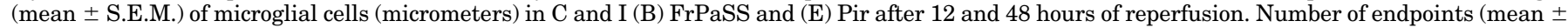

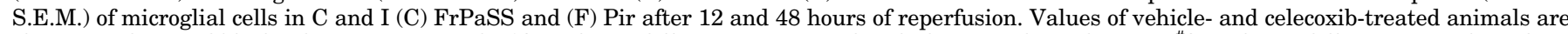

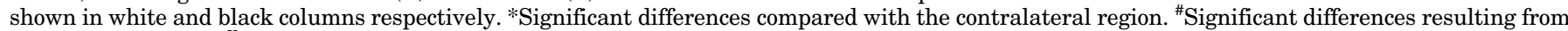

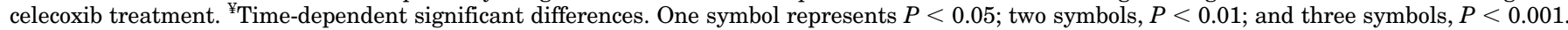
Two-way ANOVA followed by Tukey's test, $n=4$.

search for effects other than its anti-COX2 activity. These studies show a potent proapoptotic, dose-dependent effect, apparently not related to its anti-COX2 effect, in different carcinoma cell lines using concentrations ranging from 10 to $100 \mu \mathrm{M}$ (Schönthal, 2007). In contrast, celecoxib has been reported as an antiapoptotic drug in some in vivo studies, such as in myocardial infarction (Kaloustian et al., 2007), and in ICH assays (Chu et al., 2004; Sinn et al., 2007) using doses $(20 \mathrm{mg} / \mathrm{kg}$ ) that can be compared with those used in culture cells. Based on these results, we decided to explore the neuroprotective effect of celecoxib in a tMCAO model, testing a range of concentrations (20-400 $\mathrm{mg} / \mathrm{kg}$ ) that would allow us to establish its dose-dependent mortality effect. The maximal neuroprotective effect was observed at $100 \mathrm{mg} / \mathrm{kg}$, which did not elicit toxic effects and ameliorates the neurologic deficit. Thus, this concentration was used in our subsequent experiments.

Assays with different drugs have been described as unable to reduce the initial striatal ischemic core infarction in MCAO models (Carmichael, 2005). Accordingly, our TTC assays showed that celecoxib was unable to prevent the rapid necrosis in the striatum; indeed, the damage in this region was similar that seen 12 and 48 hours after injury in treated and vehicle animals. Thus, this area could be considered an infarcted area according to the criteria for ischemic lesions described elsewhere (Ejaz et al., 2013).

In contrast, in the cerebral cortex, the celecoxib postischemic treatment reduced the ischemic damage (infarct volume) based on the standard TTC assays at 48 hours. It should be noted that the red-stained tissue includes both healthy tissue and ischemic penumbra that are undistinguishable with this technique. TTC has been reported to overestimate the infarct size and may not represent irreversible cell death (Türeyen et al., 2004; Benedek et al., 2006). At a microscopic level, however, the presence of SNL areas at 12 hours of I/R can be observed when TTC still does not reveal damage in the cerebral cortex, indicating areas where neurons seem to be more vulnerable to ischemia. Therefore, these neurons are likely to have been damaged since the first stages of reperfusion. Considering the different types of damage described in the literature (Ejaz et al., 2013), we can report the existence of SNL and non-SNL areas in the cerebral cortex. Consistent with our TTC data, celecoxib treatment prevented, or at least delayed, the progression of non-SNL to SNL areas; however, long-term studies are required to determine whether the neuroprotective effect observed has a limited effect or if it maintains neuroprotection. We did not find a clear correlation between neuronal demise and number or morphology of IBA-1-positive cells (as discussed to follow); therefore, we cannot establish in what measure the neuroprotective effect of celecoxib depends on its antiapoptosis or its antiinflammatory (anti-COX-2) effect.

Why a specific area becomes an SNL area has been related to the degree of hypoperfusion during occlusion or regional susceptibility to ischemia from differences in basal neuronal activity and/or microvasculature (Hughes et al., 2010); however, neuronal vulnerability can rely on other causes, such as glutamate excitotoxicity, protein synthesis inhibition, or susceptibility to caspase activation (Nakano et al., 1990; Hossmann, 1993). It has been questioned whether SNL areas 

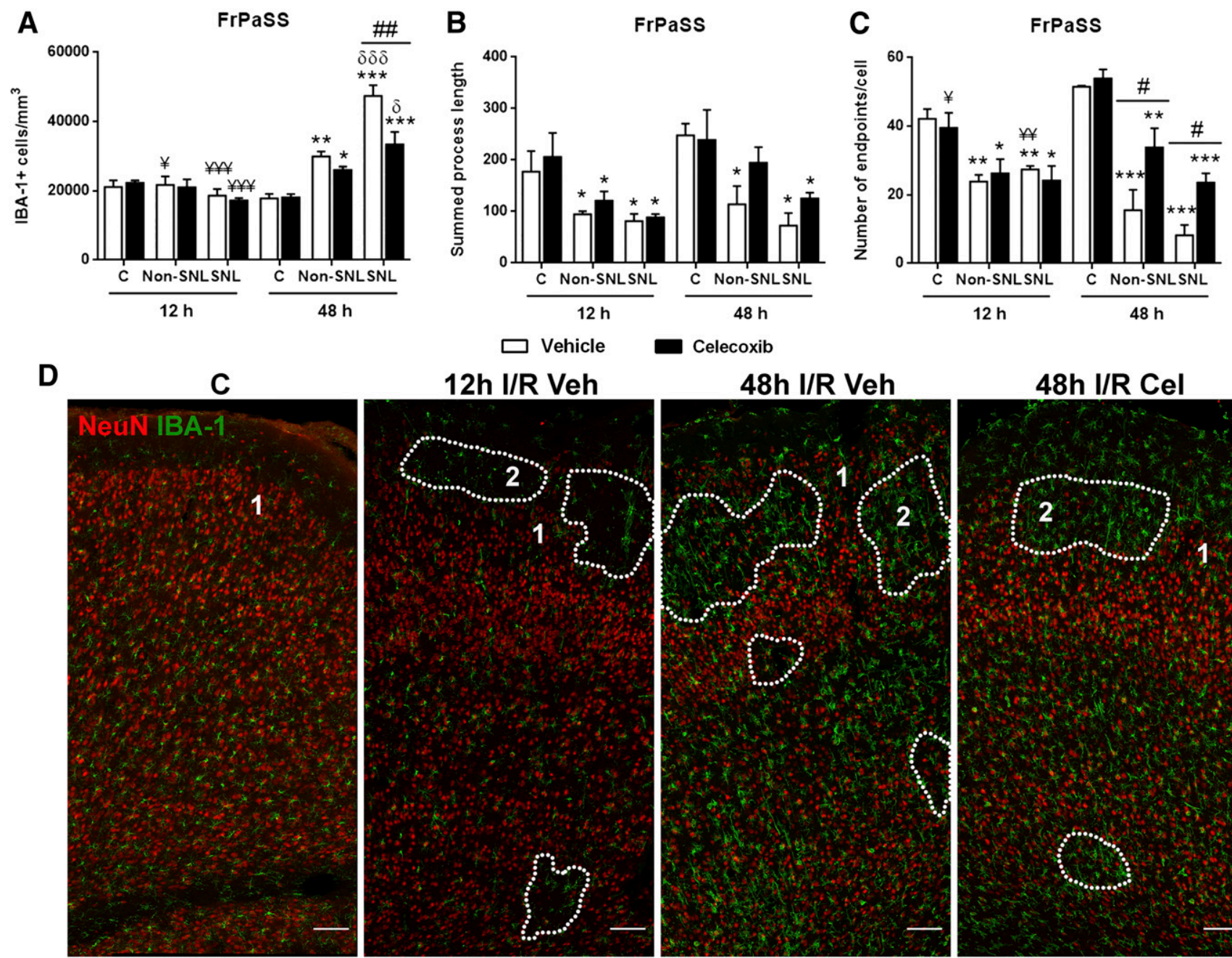

12h I/R Veh

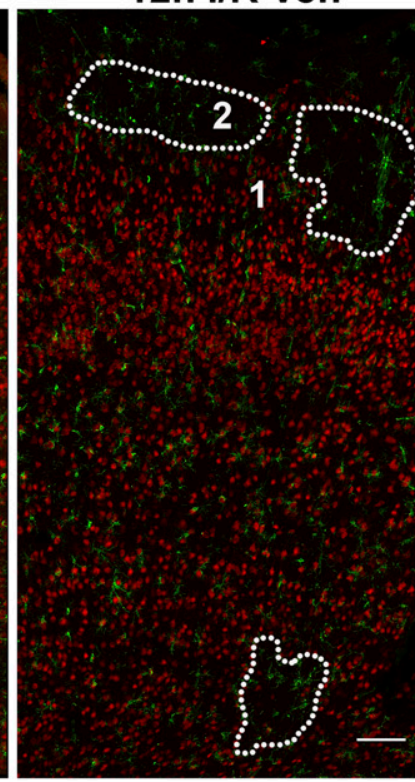

48h I/R Veh

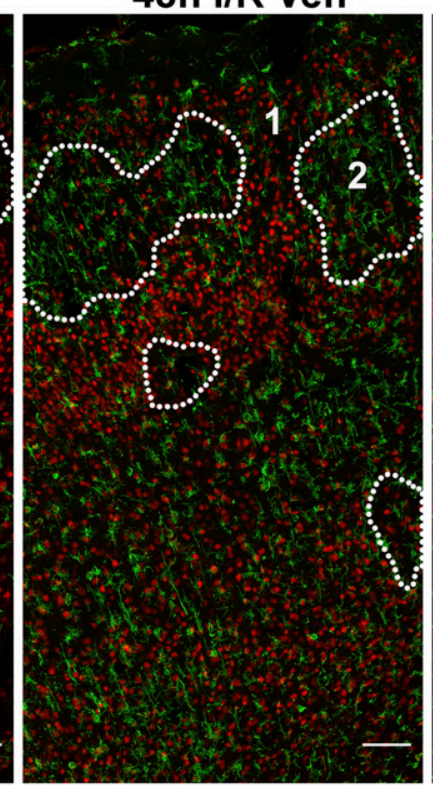

48h I/R Cel
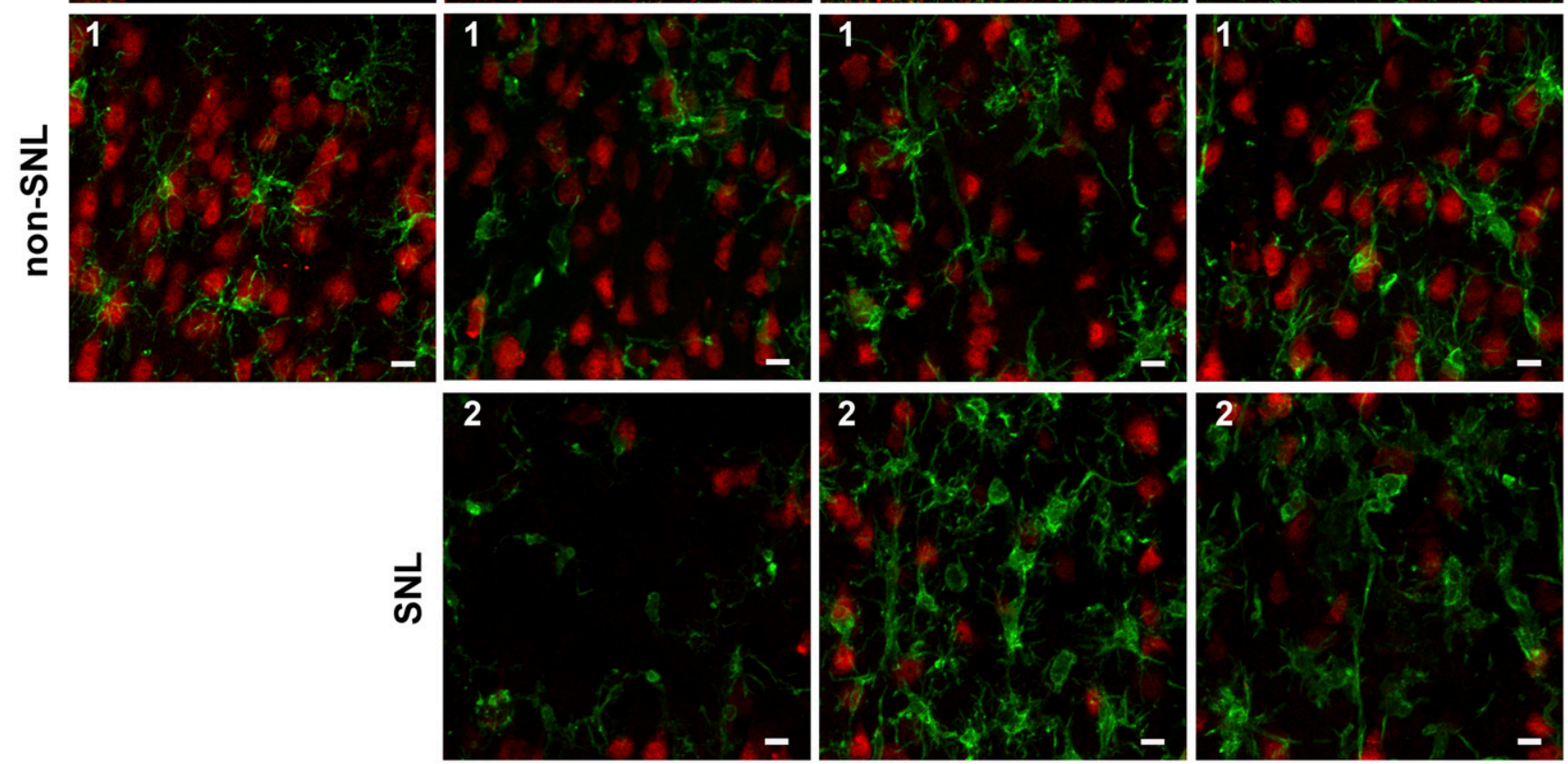

Fig. 5. Microglial density and morphology in frontoparietal cortex, somatosensory area (FrPaSS) selective neuronal loss (SNL) and non-SNL areas. (A) Microglial densities (mean \pm S.E.M.), (B) branch length (mean \pm S.E.M.) of microglia cells (micrometers), and (C) number of endpoints (mean \pm S.E.M.) of microglia cells in contralateral (C), nonselective neuronal loss (non-SNL) and SNL areas of FrPaSS after 12 and 48 hours of reperfusion. Values of vehicle- and celecoxib-treated animals are shown in white and black columns, respectively. *Significant differences with respect to the contralateral region; ${ }^{\delta}$ Significant differences between non-

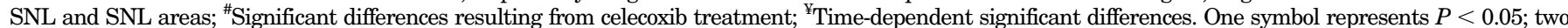
symbols, $P<0.01$; three symbols, $P<0.001$, Two-way ANOVA followed by Tukey's test, $n=4$. (D) Representative images of FrPaSS labeled with IBA1 + NeuN in the different conditions studied. The dotted line show SNL area borders. Scale bar, $100 \mu \mathrm{m}$. Details are shown in the insets. Scale bar, $10 \mu \mathrm{m}$. 

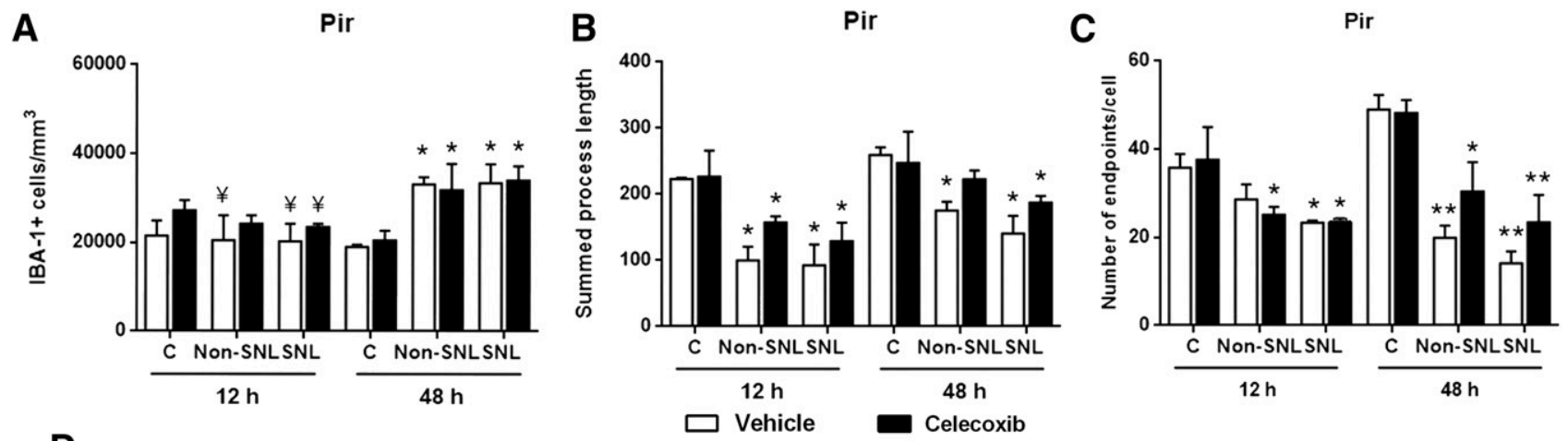

D

C

12h I/R Veh

48h I/R Veh

48h I/R Cel
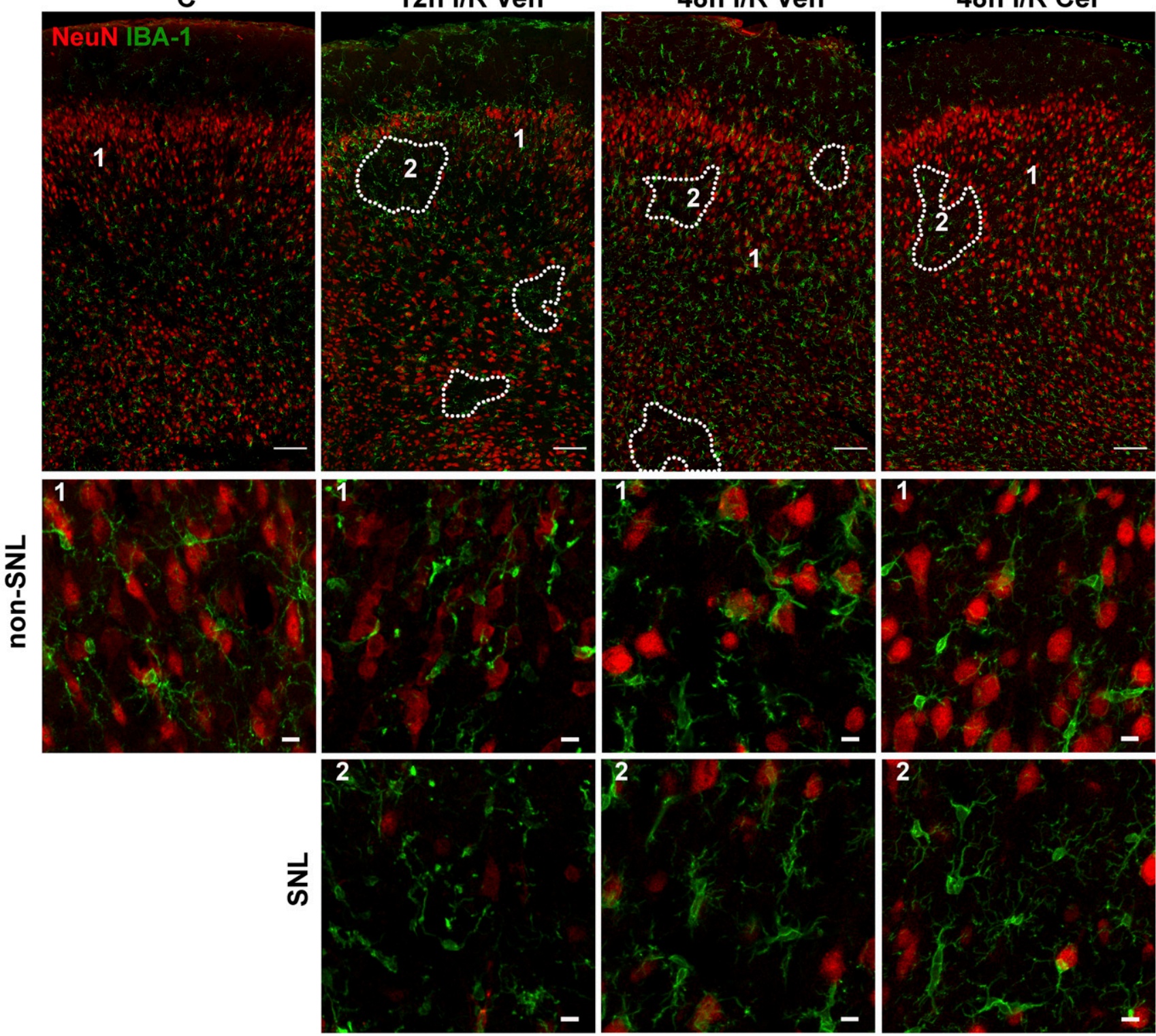

Fig. 6. Microglial density and morphology in piriform cortex (Pir) selective neuronal loss (SNL) and non-SNL areas. (A) Microglial densities (mean \pm S.E.M.). (B) Branch length (mean \pm S.E.M.) of microglia cells (micrometers) and (C) number of endpoints (mean \pm S.E.M.) of microglia cells in contralateral (C), nonselective neuronal loss (non-SNL) and SNL areas of Pir after 12 and 48 hours of reperfusion. Values of vehicle- and celecoxibtreated animals are shown in white and black columns, respectively. *Significant differences with respect to the contralateral region; ${ }^{*}$ time-dependent significant differences. One symbol represents $P<0.05$; two symbols, $P<0.01$; three symbols, $P<0.001$. Two-way ANOVA followed by Tukey's test, $n=$ 4. (D) Representative images of Pir labeled with IBA1 + NeuN in the different conditions studied. The dotted line shows SNL area borders. Scale bar, $100 \mu \mathrm{m}$. Details are shown in the insets. Scale bar, $10 \mu \mathrm{m}$. 
A

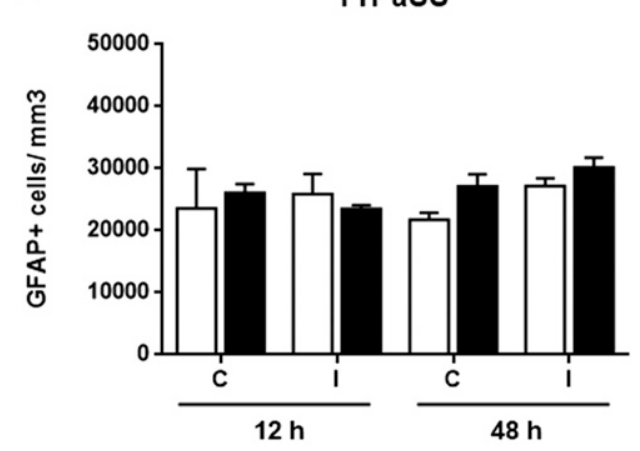

C

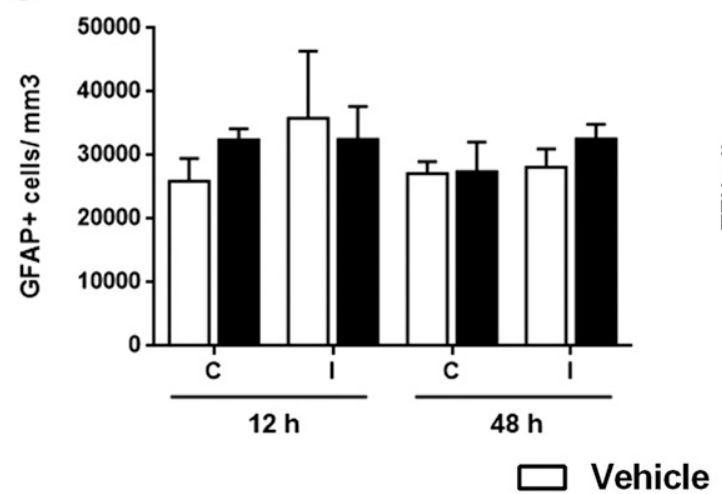

B

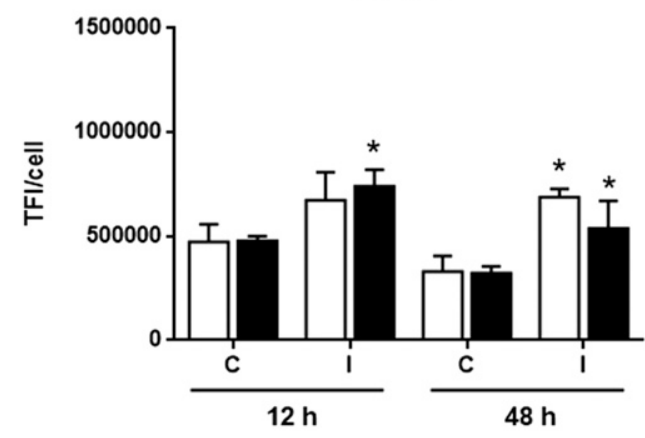

D

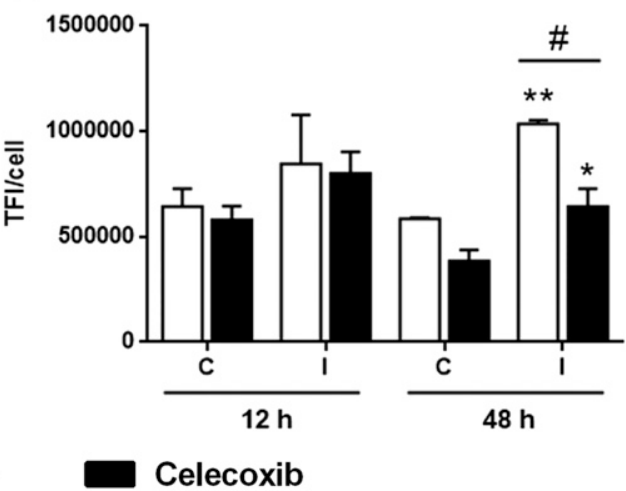

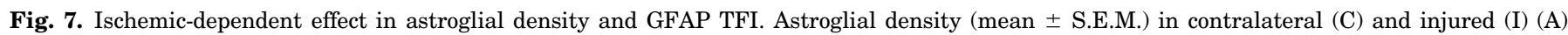

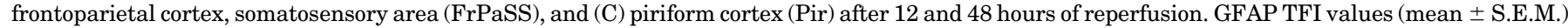

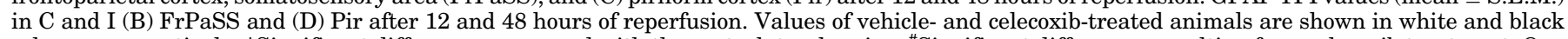

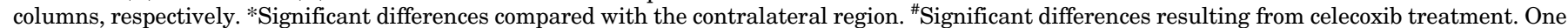
symbol represents $P<0.05$; two symbols, $P<0.01$; and three symbols, $P<0.001$. Two-way ANOVA followed by Tukey's test, $n=4$.

are determined during ischemia (and that the neuronal death would induce an increase of microglia) or the reperfusion triggered processes (such as oxygen radicals or inflammation, followed by microglia increase, which would result in neuronal death) (Baron et al., 2014). We cannot say whether the increased percentage in SNL areas mirrors an increase in new lesions along the time or the extension of previously damaged areas. What seems clear is that the presence of SNL areas distributed irregularly in the penumbra area contrast with the usually accepted idea of a core region progressing radially, which seems to support the idea of the increase in new lesions.

It must be considered that, despite the term, non-SNL areas also present some degree of neuronal demise. Thus, the significant neuronal demise in FrPaSS non-SNL areas in vehicle animals is supported by the neuroprotective treatment with celecoxib. In contrast, the lack of significant neuronal demise in Pir non-SNL areas makes it difficult to ensure neuronal demise in these areas. Our finding that celecoxib treatment increases the neuronal density of these areas also supports the idea of neuronal demise in the Pir non-SNL areas.

Is there reliability between the different techniques used to evaluate the evolving phases of infarction and the effect of neuroprotective drugs? Celecoxib-dependent neuroprotection observed with TTC after 48 hours of reperfusion fits with the data on neuronal density, when FrPaSS and Pir were analyzed as a whole without distinguishing between non-SNL and SNL areas; however, the analysis distinguishing between non-SNL and SNL areas may be biased because the celecoxib-dependent protection of neuronal loss can be underestimated. Thus, analysis by area supports the idea that celecoxib provides some degree of neuroprotection at 12 hours in SNL areas but not at 48 hours; however, at 48 hours, treatment with celecoxib decreases the ratio SNL/non-SNL (i.e., decreases the percentage of SNL area over time) (Fig. 3D). This seems to mask its neuroprotective effect when neuronal density is measured only in SNLs when celecoxib actually prevents the occurrence of SNL areas but cannot avoid the damage in the existent SNL areas. In summary, celecoxib neuroprotection can prevent neuronal mortality in non-SNL areas and delay its progress from nonSNL to SNL areas, thus explaining the neuroprotection observed at 48 hours. Pir and FrPaSS seem to show some differences in their response to ischemia, which could be a consequence of being phylogenetic and structurally different cortical areas (Hagan et al., 2012). In this regard, data on celecoxib treatment suggest the idea that non-SNL areas progress toward SNL more slowly in the Pir than in the FrPaSS (Fig. 3 ), which in turn suggests a higher neuronal vulnerability to the ischemia in the neocortex (FrPaSS) than in paleocortex (Pir).

Morphometrical analysis reveals very high level of microglial activation after 12 hours of reperfusion, without differences between SNL and non-SNL areas; however, the number of branches can still be reduced 48 hours after reperfusion, which supports the idea that further activation remains possible. These parameters did not reveal differences between 
A

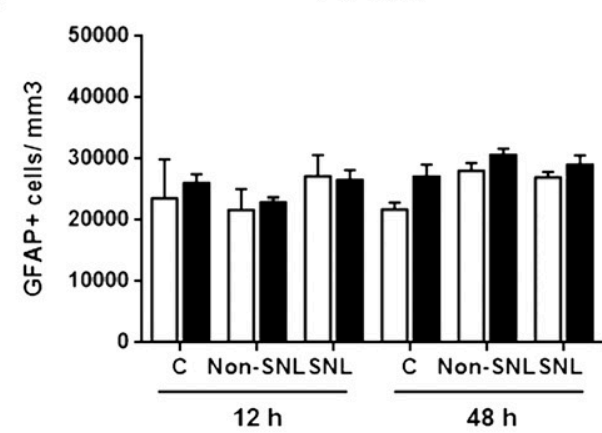

B

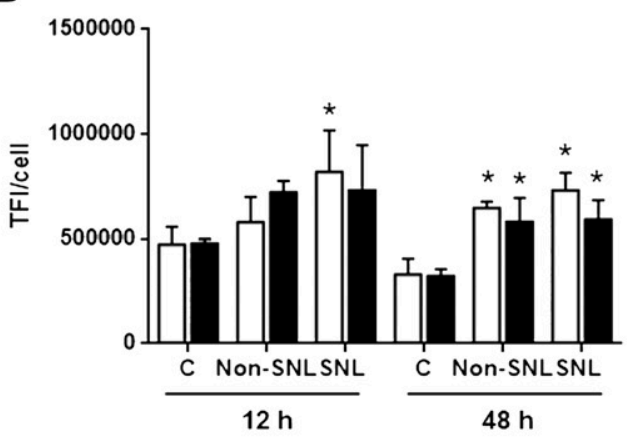

C

C
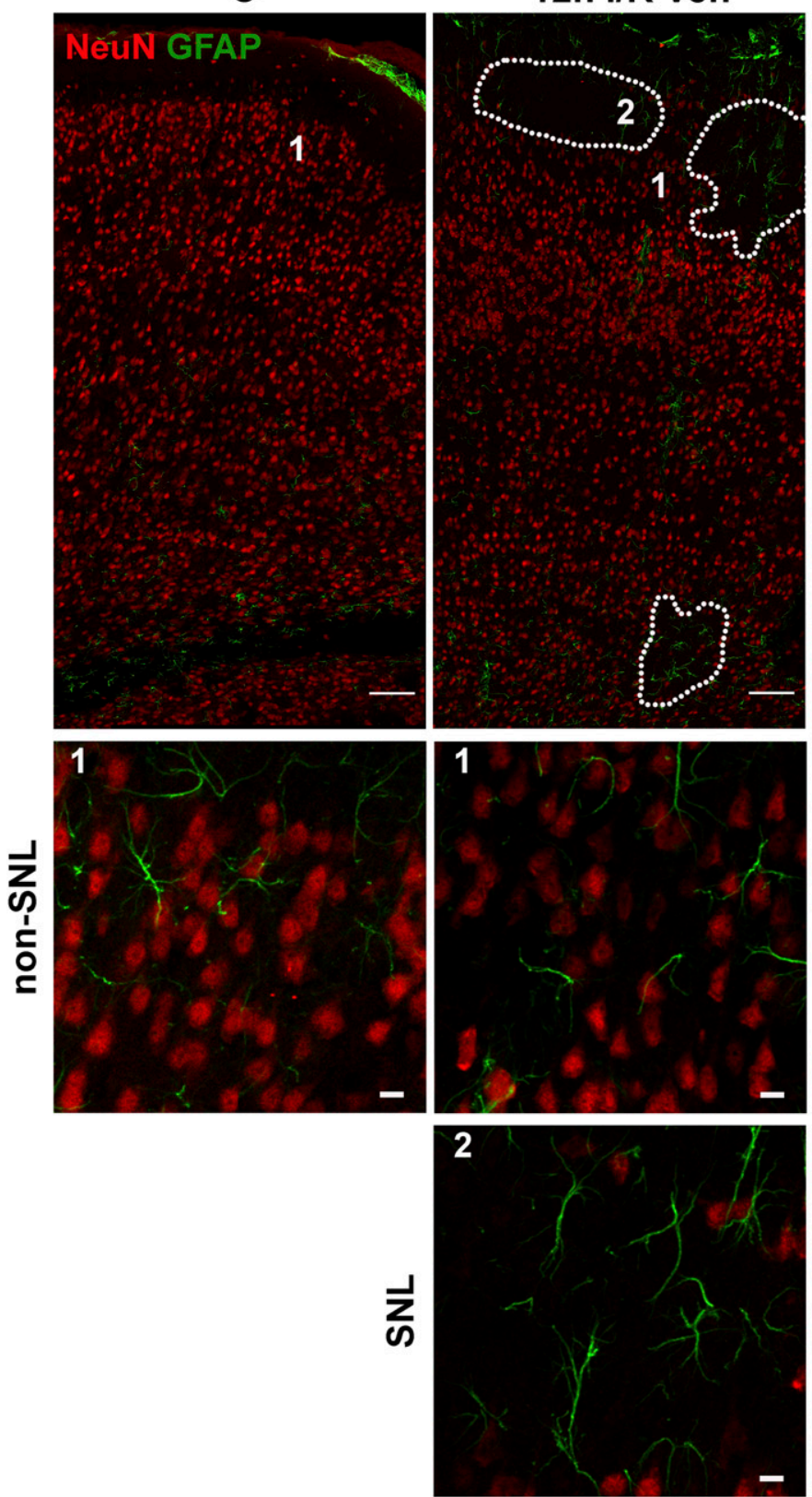

12h I/R Veh
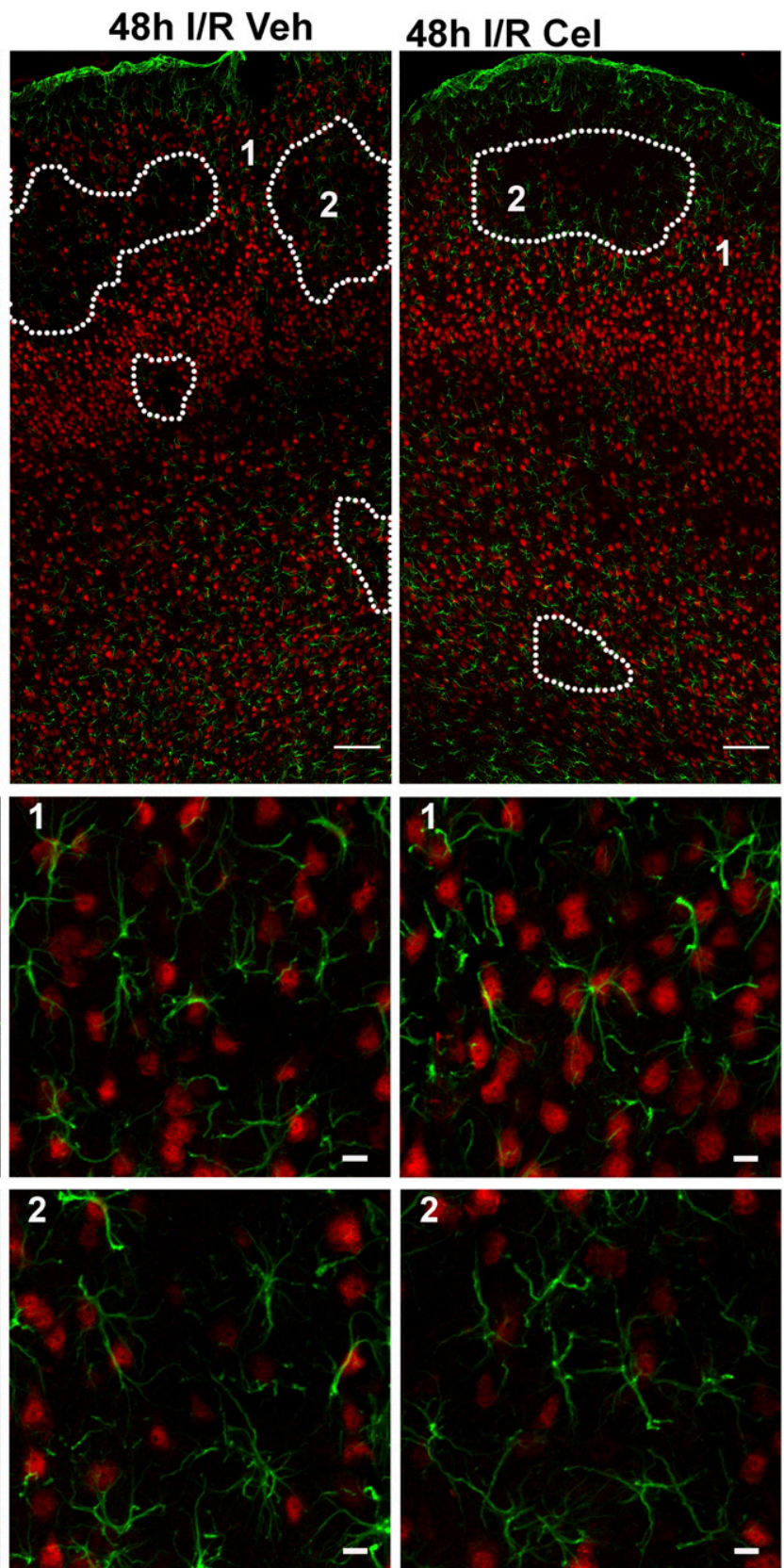

$\square$ Vehicle

Celecoxib

Fig. 8. Astroglial density and GFAP TFI in frontoparietal cortex, somatosensory area (FrPaSS) selective neuronal loss (SNL) and non-SNL areas. (A) Astroglial density (mean \pm S.E.M.) and (B) GFAP TFI values (mean \pm S.E.M.) in contralateral (C), non-SNL and SNL areas of FrPaSS after 12 and 48 hours of reperfusion. Values of vehicle- and celecoxib-treated animals are shown in white and black columns, respectively. *Significant differences compared with the contralateral region. One symbol represents $P<0.05$, two-way ANOVA, followed by Tukey's test, $n=4$. (C) Representative images of FrPaSS labeled with GFAP + NeuN in the different conditions studied. The dotted line shows SNL area borders. Scale bar, $100 \mu \mathrm{m}$. Details are shown in the insets Scale bar, $10 \mu \mathrm{m}$. 
A

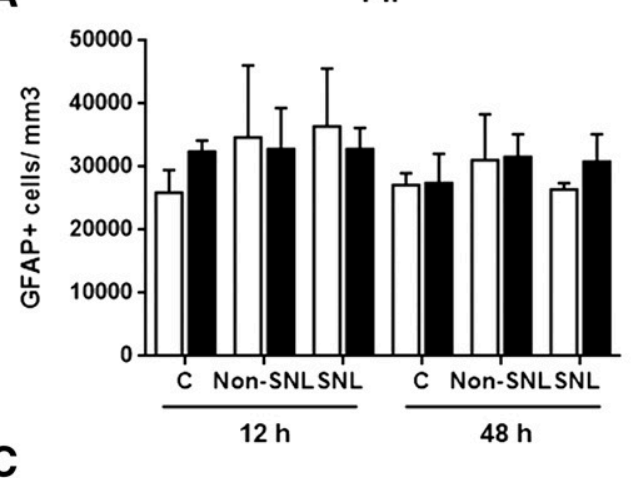

C

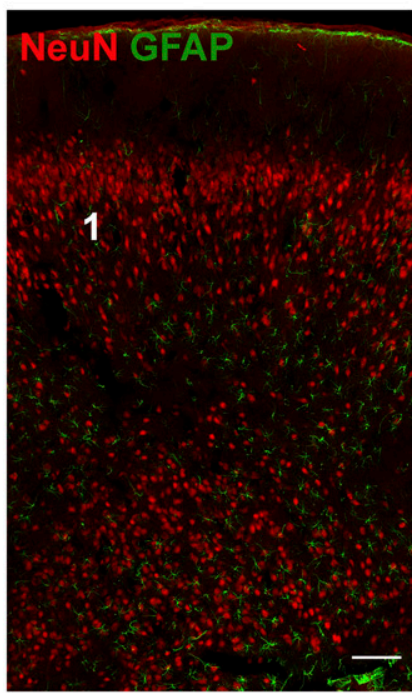

B

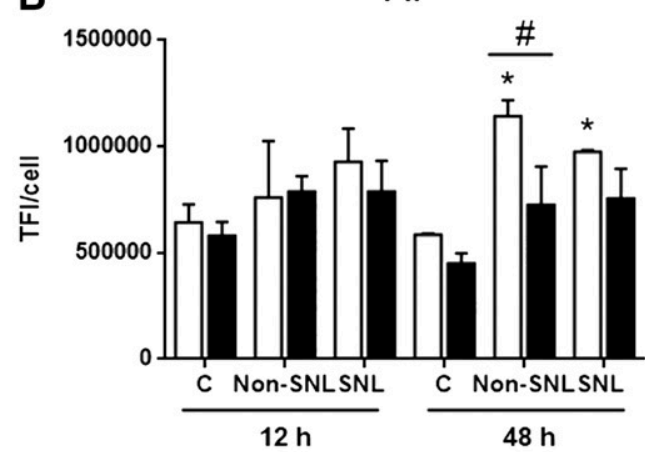

Vehicle

Celecoxib

I l/R Cel
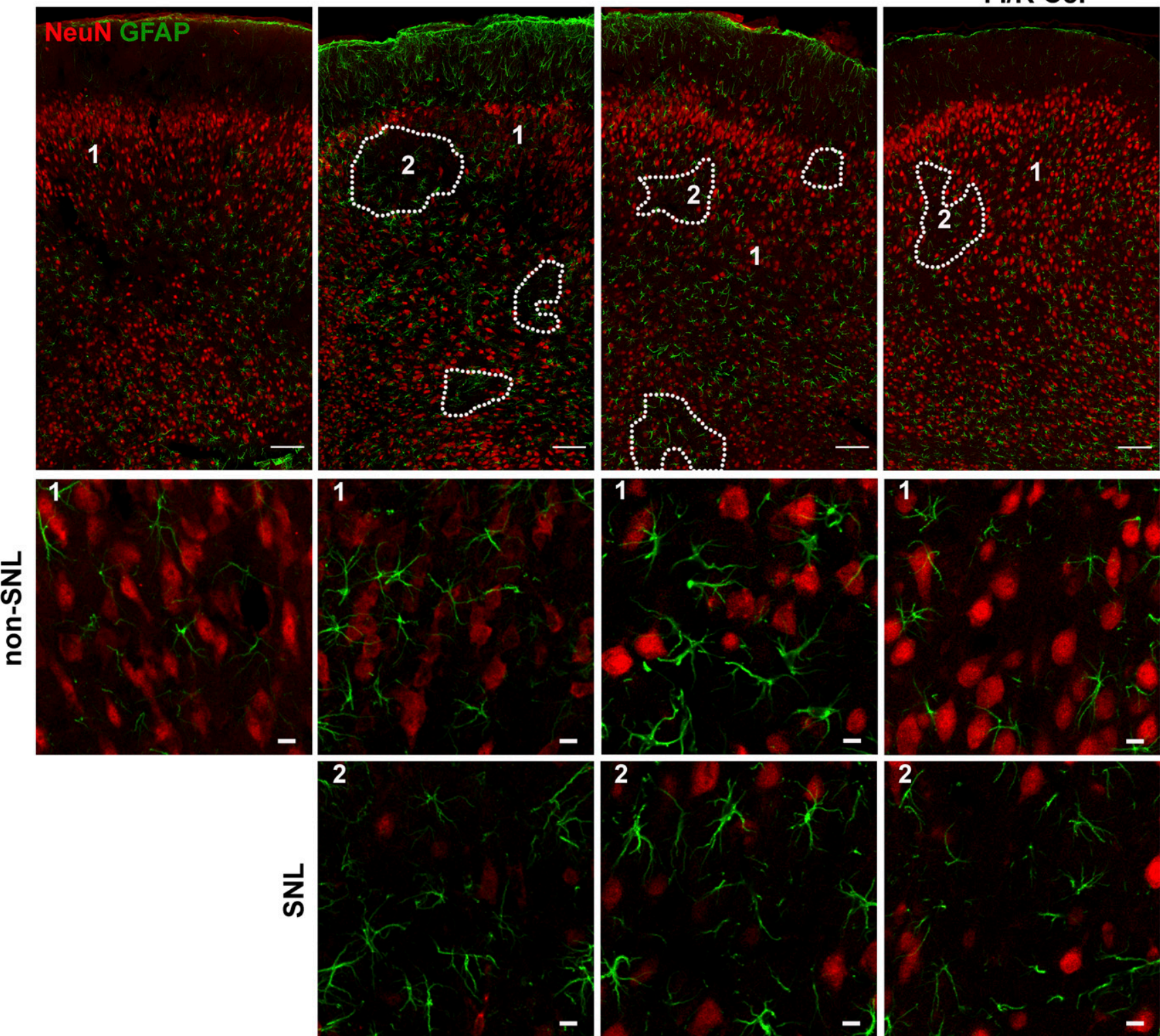

Fig. 9. Astroglial density and GFAP TFI in piriform cortex (Pir) selective neuronal loss (SNL) and non-SNL areas. (A) Astroglial density (mean \pm S.E.M.) and (B) GFAP TFI values (mean \pm S.E.M.) in contralateral (C), non-SNL and SNL areas of Pir after 12 and 48 hours of reperfusion. Values of vehicle- and celecoxib-treated animals are shown in white and black columns respectively. *Significant differences compared with the contralateral region. ${ }^{\#}$ Significant differences resulting from celecoxib treatment. One symbol represents $P<0.05$, two-way ANOVA followed by Tukey's test, $n=4$. (C) Representative images of Pir labeled with GFAP + NeuN in the different conditions studied. The dotted line shows SNL area borders. Scale bar, $100 \mu \mathrm{m}$. Details are shown in the insets Scale bar, $10 \mu \mathrm{m}$. 
Pir and FrPaSS; however, the differences detected in the microglia density between FrPaSS and Pir SNL areas after 48 hours of reperfusion suggest local brain properties, probably related to blood-brain barrier permeability, in agreement with other reports of global ischemia (Anuncibay-Soto et al., 2016). In this regard, since IBA-1 labels both microglia and infiltrated monocytes (Taylor and Sansing, 2013), we hypothesized that the permeability of FrPaSS to blood cells is greater than in Pir, which could explain the delay in SNL progress between Pir and FrPaSS as discussed already. Furthermore, celecoxib treatment reduces the density and activation of microglia in FrPaSS, and its effect is not clear in Pir, supporting the idea of different responses to celecoxib in these regions. Time-dependent differences, as well as different response to celecoxib, in the astroglial activation between FrPaSS and Pir provide further support to the idea of structural differences in response to ischemia.

In summary, we have shown that postischemic celecoxib treatment provides a neuroprotective effect in a tMCAO model (it reduces infarct volume and neurologic deficit) that seems to prevent or delay the occurrence of selective neuronal loss and reduces glial activation. Our findings support the notion of greater vulnerability to ischemia in FrPaSS than in Pir. The different effects of celecoxib on neuronal densities, microglia, and astroglial activation in these regions also support this hypothesis.

\section{Acknowledgments}

We thank Paloma González-Rodríguez for critical readings and technical support.

\section{Authorship Contributions}

Participated in research design: Santos-Galdiano, PérezRodriguez, Fernandez-Lopez.

Conducted experiments: Santos-Galdiano, Perez-Rodriguez, Anuncibay-Soto, Font-Belmonte, Ugidos, Perez-Garcia.

Contributed new reagents or analytic tools: Perez-Rodriguez, Anuncibay-Soto.

Performed data analysis: Santos-Galdiano.

Wrote or contributed to the writing of the manuscript: SantosGaldiano, Fernandez-Lopez.

\section{References}

Altman R, Bosch B, Brune K, Patrignani P, and Young C (2015) Advances in NSAID development: evolution of diclofenac products using pharmaceutical technology. Drugs 75:859-877.

Andersohn F, Schade R, Suissa S, and Garbe E (2006) Cyclooxygenase-2 selective nonsteroidal anti-inflammatory drugs and the risk of ischemic stroke: a nested case-control study. Stroke 37:1725-1730.

Anuncibay-Soto B, Pérez-Rodríguez D, Santos-Galdiano M, Font E, Regueiro-Purriños M, and Fernández-López A (2016) Post-ischemic salubrinal treatment results in a neuroprotective role in global cerebral ischemia. J Neurochem 138:295-306.

Anuncibay-Soto B, Pérez-Rodriguez D, Santos-Galdiano M, Font-Belmonte E, Ugidos IF, Gonzalez-Rodriguez P, Regueiro-Purriños M, and Fernández-López A (2018) Salubrinal and robenacoxib treatment after global cerebral ischemia: exploring the interactions between ER stress and inflammation. Biochem Pharmacol 151:26-37.

Araki E, Forster C, Dubinsky JM, Ross ME, and Iadecola C (2001) Cyclooxygenase-2 inhibitor ns-398 protects neuronal cultures from lipopolysaccharide-induced neurotoxicity. Stroke 32:2370-2375.

Arganda-Carreras I, Fernández-González R, Muñoz-Barrutia A, and Ortiz-De-Solorzano C (2010) 3D reconstruction of histological sections: application to mammary gland tissue. Microsc Res Tech 73:1019-1029.

Baron JC (2005) How healthy is the acutely reperfused ischemic penumbra? Cerebrovasc Dis 20 (Suppl 2):25-31.

Baron JC, Yamauchi H, Fujioka M, and Endres M (2014) Selective neuronal loss in ischemic stroke and cerebrovascular disease. J Cereb Blood Flow Metab 34:2-18.

Barone FC and Feuerstein GZ (1999) Inflammatory mediators and stroke: new opportunities for novel therapeutics. J Cereb Blood Flow Metab 19:819-834.

Barreto G, White RE, Ouyang Y, Xu L, and Giffard RG (2011) Astrocytes: targets for neuroprotection in stroke. Cent Nerv Syst Agents Med Chem 11:164-173.

Bederson JB, Pitts LH, Tsuji M, Nishimura MC, Davis RL, and Bartkowski H (1986) Rat middle cerebral artery occlusion: evaluation of the model and development of a neurologic examination. Stroke 17:472-476.
Benedek A, Móricz K, Jurányi Z, Gigler G, Lévay G, Hársing LG Jr, Mátyus P, Szénási G, and Albert M (2006) Use of TTC staining for the evaluation of tissue injury in the early phases of reperfusion after focal cerebral ischemia in rats. Brain Res 1116:159-165.

Bhattacharya P, Pandey AK, Paul S, Patnaik R, and Yavagal DR (2013) Aquaporin-4 inhibition mediates piroxicam-induced neuroprotection against focal cerebral ischemia/reperfusion injury in rodents. PLoS One 8:e73481.

Block F, Dihné M, and Loos M (2005) Inflammation in areas of remote changes following focal brain lesion. Prog Neurobiol 75:342-365.

Candelario-Jalil E, Alvarez D, Castañeda JM, Al-Dalain SM, Martínez-Sánchez G, Merino N, and León OS (2002) The highly selective cyclooxygenase-2 inhibitor DFU is neuroprotective when given several hours after transient cerebral ischemia in gerbils. Brain Res 927:212-215.

Candelario-Jalil E and Fiebich BL (2008) Cyclooxygenase inhibition in ischemic brain injury. Curr Pharm Des 14:1401-1418.

Carmichael ST (2005) Rodent models of focal stroke: size, mechanism, and purpose. NeuroRx 2:396-409.

Chang CH, Shau WY, Kuo CW, Chen ST, and Lai MS (2010) Increased risk of stroke associated with nonsteroidal anti-inflammatory drugs: a nationwide case-crossover study. Stroke 41:1884-1890.

Chu K, Jeong SW, Jung KH, Han SY, Lee ST, Kim M, and Roh JK (2004) Celecoxib induces functional recovery after intracerebral hemorrhage with reduction of brain edema and perihematomal cell death. J Cereb Blood Flow Metab 24:926-933.

Creutzfeldt CJ, Holloway RG, and Walker M (2012) Symptomatic and palliative care for stroke survivors. J Gen Intern Med 27:853-860.

Doré S, Otsuka T, Mito T, Sugo N, Hand T, Wu L, Hurn PD, Traystman RJ, and Andreasson K (2003) Neuronal overexpression of cyclooxygenase-2 increases cerebral infarction. Ann Neurol 54:155-162.

Ejaz S, Williamson DJ, Ahmed T, Sitnikov S, Hong YT, Sawiak SJ, Fryer TD, Aigbirhio FI, and Baron JC (2013) Characterizing infarction and selective neuronal loss following temporary focal cerebral ischemia in the rat: a multi-modality imaging study. Neurobiol Dis 51:120-132.

Emmrich JV, Ejaz S, Neher JJ, Williamson DJ, and Baron JC (2015) Regional distribution of selective neuronal loss and microglial activation across the MCA territory after transient focal ischemia: quantitative versus semiquantitative systematic immunohistochemical assessment. J Cereb Blood Flow Metab 35:20-27.

Fluri F, Schuhmann MK, and Kleinschnitz C (2015) Animal models of ischemic stroke and their application in clinical research. Drug Des Devel Ther 9:3445-3454.

Galea J and Brough D (2013) The role of inflammation and interleukin-1 in acute cerebrovascular disease. J Inflamm Res 6:121-128.

Garcia JH, Lassen NA, Weiller C, Sperling B, and Nakagawara J (1996) Ischemic stroke and incomplete infarction. Stroke 27:761-765.

Gundersen HJ, Bagger P, Bendtsen TF, Evans SM, Korbo L, Marcussen N, Møller A, Nielsen K, Nyengaard JR, Pakkenberg B, et al. (1988) The new stereological tools: disector, fractionator, nucleator and point sampled intercepts and their use in pathological research and diagnosis. APMIS 96:857-881.

Hagan CE, Bolon B, and Keene CD (2012) Nervous system, in Comparative Anatomy and Histology (Treuting PM, Dintzis S, Liggitt D, and Frevert CW eds) pp 339-394, Elsevier Inc, Amsterdam, Netherlands.

Heiss WD and Rosner G (1983) Functional recovery of cortical neurons as related to degree and duration of ischemia. Ann Neurol 14:294-301.

Heit JJ and Wintermark M (2016) Perfusion computed tomography for the evaluation of acute ischemic stroke: strengths and pitfalls. Stroke 47:1153-1158.

Hossmann KA (1993) Disturbances of cerebral protein synthesis and ischemic cell death. Prog Brain Res 96:161-177.

Hughes JL, Beech JS, Jones PS, Wang D, Menon DK, and Baron JC (2010) Mapping selective neuronal loss and microglial activation in the salvaged neocortical penumbra in the rat. Neuroimage 49:19-31.

Ito D, Tanaka K, Suzuki S, Dembo T, and Fukuuchi Y (2001) Enhanced expression of Iba1, ionized calcium-binding adapter molecule 1, after transient focal cerebral ischemia in rat brain. Stroke 32:1208-1215.

Ji K and Tsirka SE (2012) Inflammation modulates expression of laminin in the central nervous system following ischemic injury. J Neuroinflammation 9:159.

Kaloustian S, Wann BP, Bah TM, Falcao S, Dufort AM, Ryvlin P, Godbout R, and Rousseau G (2007) Celecoxib after the onset of reperfusion reduces apoptosis in the amygdala. Apoptosis 12:1945-1951.

Karperien A, Ahammer H, and Jelinek HF (2013) Quantitating the subtleties of microglial morphology with fractal analysis. Front Cell Neurosci 7:3.

Leahy KM, Ornberg RL, Wang Y, Zweifel BS, Koki AT, and Masferrer JL (2002) Cyclooxygenase-2 inhibition by celecoxib reduces proliferation and induces apoptosis in angiogenic endothelial cells in vivo. Cancer Res 62:625-631.

Lee SH, Park HK, Ryu WS, Lee JS, Bae HJ, Han MK, Lee YS, Kwon HM, Kim CK, Park ES, et al. (2013) Effects of celecoxib on hematoma and edema volumes in primary intracerebral hemorrhage: a multicenter randomized controlled trial. Eur $J$ Neurol 20:1161-1169.

López-Villodres JA, De La Cruz JP, Muñoz-Marin J, Guerrero A, Reyes JJ, and González-Correa JA (2012) Cytoprotective effect of nonsteroidal antiinflammatory drugs in rat brain slices subjected to reoxygenation after oxygenglucose deprivation. Eur J Pharm Sci 45:624-631.

Moore RA, Derry S, Makinson GT, and McQuay HJ (2005) Tolerability and adverse events in clinical trials of celecoxib in osteoarthritis and rheumatoid arthritis: systematic review and meta-analysis of information from company clinical trial reports. Arthritis Res Ther 7:R644-R665.

Morrison HW and Filosa JA (2013) A quantitative spatiotemporal analysis of microglia morphology during ischemic stroke and reperfusion. J Neuroinflammation 10:4

Nakano S, Kogure K, and Fujikura H (1990) Ischemia-induced slowly progressive neuronal damage in the rat brain. Neuroscience 38:115-124.

Nogawa S, Zhang F, Ross ME, and Iadecola C (1997) Cyclo-oxygenase-2 gene expression in neurons contributes to ischemic brain damage. $J$ Neurosci 17: 2746-2755. 
Pyrko P, Kardosh A, and Schönthal AH (2008) Celecoxib transiently inhibits cellular protein synthesis. Biochem Pharmacol 75:395-404.

Reglodi D, Tamás A, and Lengvári I (2003) Examination of sensorimotor performance following middle cerebral artery occlusion in rats. Brain Res Bull 59:459-466.

Schönthal AH (2007) Direct non-cyclooxygenase-2 targets of celecoxib and their potential relevance for cancer therapy. $\mathrm{Br} J$ Cancer 97:1465-1468.

Senda DM, Franzin S, Mori MA, de Oliveira RM, and Milani H (2011) Acute, postischemic sensorimotor deficits correlate positively with infarct size but fail to predict its occurrence and magnitude after middle cerebral artery occlusion in rats. Behav Brain Res 216:29-35.

Shichita T, Ago T, Kamouchi M, Kitazono T, Yoshimura A, and Ooboshi H (2012) Novel therapeutic strategies targeting innate immune responses and early inflammation after stroke. J Neurochem 123 (Suppl 2):29-38.

Shientag LJ, Wheeler SM, Garlick DS, and Maranda LS (2012) A therapeutic dose of ketoprofen causes acute gastrointestinal bleeding, erosions, and ulcers in rats. $J$ Am Assoc Lab Anim Sci 51:832-841.

Sinn DI, Lee ST, Chu K, Jung KH, Song EC, Kim JM, Park DK, Kim M, and Roh JK (2007) Combined neuroprotective effects of celecoxib and memantine in experimental intracerebral hemorrhage. Neurosci Lett 411:238-242.

Sofroniew MV and Vinters HV (2010) Astrocytes: biology and pathology. Acta Neuropathol 119:7-35.

Taylor RA and Sansing LH (2013) Microglial responses after ischemic stroke and intracerebral hemorrhage. Clin Dev Immunol 2013:746068.

Türeyen K, Vemuganti R, Sailor KA, and Dempsey RJ (2004) Infarct volume quantification in mouse focal cerebral ischemia: a comparison of triphenyltetrazolium chloride and cresyl violet staining techniques. J Neurosci Methods 139:203-207.

Ugidos IF, Santos-Galdiano M, Pérez-Rodríguez D, Anuncibay-Soto B, FontBelmonte E, López DJ, Ibarguren M, Busquets X, and Fernández-López A (2017) Neuroprotective effect of 2 -hydroxy arachidonic acid in a rat model of transient middle cerebral artery occlusion. Biochim Biophys Acta Biomembr $\mathbf{1 8 5 9}$ (9 Pt B):1648-1656.

Vane JR, Bakhle YS, and Botting RM (1998) Cyclooxygenases 1 and 2. Annu Rev Pharmacol Toxicol 38:97-120.

Varas-Lorenzo C, Riera-Guardia N, Calingaert B, Castellsague J, Pariente A, Scotti L, Sturkenboom M, and Perez-Gutthann S (2011) Stroke risk and NSAIDs: a systematic review of observational studies. Pharmacoepidemiol Drug Saf 20:1225-1236.

Walker FR, Beynon SB, Jones KA, Zhao Z, Kongsui R, Cairns M, and Nilsson M (2014) Dynamic structural remodelling of microglia in health and disease: a review of the models, the signals and the mechanisms. Brain Behav Immun 37:1-14.

Wang Q, Tang XN, and Yenari MA (2007) The inflammatory response in stroke. $J$ Neuroimmunol 184:53-68.

Wang Z, Huang W, and Zuo Z (2014) Perioperative aspirin improves neurological outcome after focal brain ischemia possibly via inhibition of Notch 1 in rat. $J$ Neuroinflammation 11:56.

Wilhelmsson U, Bushong EA, Price DL, Smarr BL, Phung V, Terada M, Ellisman $\mathrm{MH}$, and Pekny M (2006) Redefining the concept of reactive astrocytes as cells that remain within their unique domains upon reaction to injury. Proc Natl Acad Sci USA 103:17513-17518.

Ye Z, Wang N, Xia P, Wang E, Liao J, and Guo Q (2013) Parecoxib suppresses CHOP and Foxo1 nuclear translocation, but increases GRP78 levels in a rat model of focal ischemia. Neurochem Res 38:686-693.

Yenari MA, Kauppinen TM, and Swanson RA (2010) Microglial activation in stroke: therapeutic targets. Neurotherapeutics 7:378-391.

Address correspondence to: Dr. Arsenio Fernández-López, Área de Biología Celular, Instituto de Biomedicina, Universidad de León, 24071 León, Spain. E-mail: aferl@unileon.es 